TI 2018-008/III

Tinbergen Institute Discussion Paper

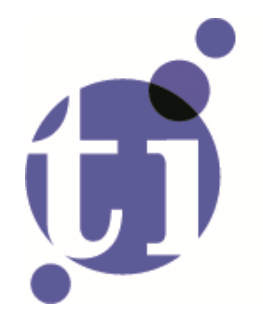

\title{
An Epidemiological Model of Crisis Spread Across Sectors in The United States
}

Eva (E.F.) Janssens ${ }^{1}$

Robin (R.) Lumsdaine ${ }^{2}$

Sebastiaan (S.H.L.C.G.) Vermeulen ${ }^{3}$

1: Tinbergen Institute; Econometrics Institute, Erasmus University of Rotterdam

2: Kogod School of Business, American University; Econometrics Institute, Erasmus University of Rotterdam;National Bureau of Economic Research and Center for Financial Stability; Tinbergen Institute, The Netherlands

3: Tinbergen Institute; Econometrics Institute, Erasmus University of Rotterdam 
Tinbergen Institute is the graduate school and research institute in economics of Erasmus University Rotterdam, the University of Amsterdam and VU University Amsterdam.

Contact: discussionpapers@tinbergen.nl

More TI discussion papers can be downloaded at the Tinbergen Site

Tinbergen Institute has two locations:

Tinbergen Institute Amsterdam

Gustav Mahlerplein 117

1082 MS Amsterdam

The Netherlands

Tel.: +31(0)20 5984580

Tinbergen Institute Rotterdam

Burg. Oudlaan 50

3062 PA Rotterdam

The Netherlands

Tel.: +31(0)10408 8900 


\title{
An Epidemiological Model of Crisis Spread Across Sectors in the United States
}

\author{
Eva F. Janssens ${ }^{(\dagger, \ddagger)}$, Robin L. Lumsdaine ${ }^{(*, \dagger, \ddagger)}$ \\ and Sebastiaan H.L.C.G. Vermeulen ${ }^{(\dagger, \$)}$ \\ (†) Econometrics Institute, Erasmus University of Rotterdam \\ (*) Kogod School of Business, American University, \\ National Bureau of Economic Research and Center for Financial Stability \\ (†) Tinbergen Institute \\ January 12, 2018
}

\begin{abstract}
This paper develops a discrete-time epidemiological model for the spread of crises across sectors in the United States for the period 1952-2015. It is the first to use an epidemiological approach with macroeconomic (Flow of Funds) data. An extension of the usual one-period Markov model to a two-period setting incorporates the concept of downturns that may either precede a crisis or from which the sector may recover and avert a crisis. The results indicate that the nonfinancial business and private depository institutions \& money market mutual funds sectors are highly contagious while the monetary authority is the least contagious.
\end{abstract}

Keywords: Flow of Funds, economic downturns, Susceptible-Infected-Removed (SIR), contagion, epidemiology

JEL classification codes: E37, E32, E01, G01

\footnotetext{
* Acknowlegements: We would like to thank Laurens Weijs and Beert van den Hoorn for their contributions on a very early precursor to this paper and Dick van Dijk, Philip Hans Franses and Ioannis Spyridopoulos for comments on an earlier draft. We also thank seminar participants at Cass Business School and the Nederlandse Economendag conference for their valuable suggestions. Any errors are our own. Lumsdaine is grateful for the generous hospitality during various visits to CREATES, Erasmus University Rotterdam, and the University of Portsmouth in contributing to the completion of this project.

Contact information:

Eva F. Janssens: Tinbergen Institute Amsterdam, Gustav Mahlerplein 117, 1082 MS Amsterdam, The Netherlands, e-mail: e.f.janssens@tinbergen.nl

Robin L. Lumsdaine (corresponding author): Kogod School of Business, American University, 4400 Massachusetts Avenue, NW, Washington, DC 20016-8044, e-mail: robin.lumsdaine@american.edu Sebastiaan H.L.C.G. Vermeulen: Tinbergen Institute Rotterdam, Burgemeester Oudlaan 50, 3062 PA Rotterdam, The Netherlands, e-mail: vermeulen@ese.eur.nl
} 


\section{Introduction}

Economists and policymakers have long relied on health-related metaphors to describe the state of the macroeconomy (Boers, 1997; Hooker, 2014). Through these metaphors, a variety of other health-related terms have entered the economic lexicon. For example, much like a disease, economic shocks are described as being transmitted and propagated (spread or contagion) and financial crises are referred to as epidemics (Shiller, 2008, 2014).

The aftermath of the recent financial crisis has seen a large increase in the use of network analysis to characterize shock transmission and the ensuing effect on a system (e.g., of financial market participants, banks, sovereign nations). In conjunction with these investigations, researchers have begun to employ methods from disciplines such as epidemiology, engineering, biology and ecology to study the transmission of financial crises, e.g., Demiris, Kypraios, and Smith (2014); Gai, Haldane, and Kapadia (2011); Haldane and May (2011). As discussed by Haldane and May (2011), the theoretical case for focusing preventive action on super-spreaders within a network — often identified by means of "centrality" — to limit the potential for system-wide spread is especially relevant within financial networks. Specifically, epidemiological models that have proven effective in managing and preventing pandemics should be of interest to economists hoping to similarly curtail the spread of potentially damaging shocks to the economy (Peckham, 2013). From a policy perspective, therefore, an epidemiological model of the macroeconomy might help in the prevention and management of future crises. In addition, such a model might provide guidance to policymakers seeking to apply limited resources for the purpose of providing economic stimulus.

Epidemiological models have been used in a number of cases to consider specific applications in networks with a large number of participants, such as transmission in a network of European banks (Glasserman \& Young, 2015; Toivanen, 2013) or the transmission of currency crises using exchange rate data (Demiris et al., 2014). In part because of the focus on financial crises, these models typically have been estimated on large networks with high frequency data. One paper that uses lower frequency data in an epidemiological context is Garas, Argyrakis, Rozenblat, Tomassini, and Havlin (2010), where international trade data are used to generate an economic network for 
the world economy and the network weights are then used as input parameters in an epidemiological model. Their subsequent analysis uses probabilistic simulations rather than explicitly specifying crisis definitions and hence differs substantially from the approach we use in this paper.

Despite these developments, epidemiological models have not been used to consider the transmission of deterioration in broad macroeconomic sectors, as occurred with the financial crisis of 2007-8 (i.e., a large increase in mortgage defaults in the household sector evolved into a crisis for the entire United States economy, and subsequently the global economy). In this paper, we construct an epidemiological model to describe how a crisis that starts in one sector spreads to other sectors of the US economy. For this purpose, we use the data on the Financial Accounts of the United States - also known as the Federal Reserve's Flow of Funds data set - a comprehensive accounting of the net worth of sectors of the US economy over a long time period. ${ }^{1}$ Because of the low (quarterly) frequency of the Flow of Funds data, rather than use a continuous model we adapt the epidemiological model by Kermack and McKendrick (1927) to a discrete version, which is appropriate as both the continuous and discrete version are representations of the same Markov chain.

In a sense, the models we propose are somewhat related to Markov switching models (Hamilton, 1989), due to their specification in terms of Markov chains. In the epidemiological model, however, the states of a sector are assumed to be observed, whereas states are determined endogenously in a Markov switching model. Furthermore, transition probabilities between states in the epidemiological models are time varying and depend on the states of all other sectors, a feature which is essential for capturing the concepts of contagion and spread of crises. Finally, while in principle a Markov switching model can accommodate any size system of equations, in practice a large system is rather unwieldy. For these reasons, we take a novel approach that likens transmission of deteriorating macroeconomic conditions to that of disease spread.

This research is the first (i) to use the Flow of Funds data set in an epidemiological model and (ii) to use this model to draw inference regarding the spread of crises

\footnotetext{
${ }^{1}$ One of the sectors in the Flow of Funds data is "Rest of the World" - hence the Flow of Funds data represents a closed macroeconomic system.
} 
through macroeconomic sectors of the US economy. The results indicate that: (a) The Private Depository Institutions and Money Market Mutual Funds sector is highly contagious, in that a crisis in this sector will likely infect four additional sectors (out of 14) and in particular results in a 38\% likelihood of the Monetary Authority sector becoming infected; (b) The Nonfinancial Business sectors (both Corporate and Noncorporate) are also highly contagious; and (c) In contrast (and perhaps reassuringly), the Monetary Authority sector is the least contagious, and although it has a high probability of entering a downturn, it also has very high one-period recovery probabilities from both downturn and crisis states (above $80 \%$ in both cases). These findings thus provide empirical support for the decisions made regarding the allocation of resources during the most recent financial crisis. For example, two of the major policy responses in the United States in October 2008 were the Federal Reserve's Money Market Investor Funding Facility (MMIFF) and the passage of the Troubled Asset Relief Program (TARP), which respectively targeted the Private Depository Institutions and Money Market Mutual Funds sector and the Nonfinancial Business sectors.

The paper is organized as follows. The next section contains a brief description of epidemiological models and relevant literature in order to present the precise specifications used, as well as a discussion of the relationship of epidemiological models to other econometric models used to analyze the transmission of shocks. Section III describes the Flow of Funds data and provides descriptive statistics. Section IV presents the results. Section V concludes. Associated with this paper is also a Supplementary Online Appendix, where one can find additional details on the maximum likelihood estimation of the models, summary statistics of the data, parameter estimates and a robustness section.

\section{An Epidemiological Model of the US Economy}

\section{General introduction to epidemiological models}

There is a large strand of literature on epidemiology, which has led to a considerable collection of epidemiological models. These models can be divided into stochastic and deterministic models. Stochastic models allow for random variation in one or more inputs over time, such as variation in exposure risk. Stochastic modeling is used when these random variations are deemed important. Alternatively, one might 
consider deterministic modeling. This latter class of models can again be subdivided into continuous and discrete models.

The first deterministic continuous model, called the Susceptible - Infected - Removed (SIR) model, was introduced by Kermack and McKendrick (1927). In a continuous model, transitions between the different categories (S, I, R in this case) are commonly represented by a set of differential equations. Since the original SIR model, a number of different modifications and extensions have emerged. These include a variety of SI models, where there is no 'removed' class as individuals of the population neither become immune nor die, or SEIR models, where E stands for 'exposed, but not infectious', implying there is a phase in the disease where one is infected, but not yet infectious. Additional extensions have been made to take into account nonhomogeneity across groups, that is, by allowing different subgroups to have different transmission rates. ${ }^{2}$

An alternative approach to continuous representations with differential equations is the use of deterministic discrete models; these are generally more flexible than continuous models in allowing for nonhomogeneity. For example, Becker and Angulo (1981) consider a chain-binomial epidemiological model for the spread of the disease and use maximum likelihood estimation to obtain their parameters. The models this paper employs fall in the category of deterministic discrete models.

This section proposes two different epidemiological models to characterize the transmission of crises across economic sectors of the US economy. The first is a discrete model that builds on the work of both Lajmanovich and Yorke (1976) and Becker and Angulo (1981), adapted to the Flow of Funds context by allowing different sectors to have different contact rates (i.e., different within-sector rates of infection and different between-sector rates of contagion). This baseline model will be referred to as the dichotomous model, a reference to the way in which a crisis is defined in this model (described below). A more realistic extension of this first model, allowing for downturns and recoveries via a two-period Markov specification, will be called the two-level model; we consider two variants, one in which recovery from a crisis is immediate and another where recovery is more gradual and includes the possibility of a relapse. Each

\footnotetext{
${ }^{2}$ For example, Lajmanovich and Yorke (1976) proposed and analyzed an 8-group SI model for gonococcal infections where the groups differ by gender and level of sexual activity and are allowed to have different contact rates.
} 
model is described in turn, by first introducing the definition of a crisis each uses. At the end of the section, the relationship between these proposed epidemiological models and two other econometric models is presented, namely, the vector autoregressive model (VAR) and the Markov switching model of Hamilton (1989). We also discuss how these epidemiological models relate to the debate in the literature on methods for dating business cycles (Hamilton, 2003; Harding \& Pagan, 2003a, 2003b) that compares the Markov switching model to a nonparametric algorithm.

\section{Sector specific crises}

The probability of entering a "crisis" is estimated using sector-level data for the models we employ. However, there are no data available on sector-level crises per se. Therefore, we initially define a sector-level crisis as a period of decline in sectoral net worth (total assets minus total liabilities). This is described in more detail in the Data section. Based on this definition, a sector is either (a) in crisis, or (b) not in crisis, leading to a dichotomous specification of the model. Later on, we consider alternative definitions, such as two consecutive periods of decline (analogous to the macroeconomic definition that a country is in recession following two consecutive periods of decline in gross domestic product (GDP)) as a way of introducing a richer type of model. A comparison of the different definitions using a simple example is contained in Table 1; we will refer to this table again when the other definitions are introduced.

[Insert Table 1]

\section{Dichotomous model}

For the dichotomous model we will use the following assumptions, similar to those of Becker and Angulo (1981) but adapted to our specific application.

1. The world consists of a pre-specified constant number of sectors, $N$. Each sector has its own recovery rate and rate of interaction with other sectors. It is also possible to aggregate several sectors together, if there are reasons to believe they are homogeneous (i.e., have the same recovery rate and rate of interaction with other sectors), and thus reduce the model dimension. 
2. A sector can be in one of two states: (1) susceptible (i.e., not infected), or (2) infectious. Unlike in many epidemiological models of disease, in the economic context, a sector cannot become immune (i.e., there is no "removed" state).

3. A sector is susceptible as long as the growth of its net worth is positive. A sector is infectious if it has had one period of negative net worth growth, where net worth is defined as total assets - total liabilities. It stays infectious until its net worth growth turns positive again, at which point it recovers (i.e. is susceptible again). For a numerical example, consider the third column of Table 1.

4. A crisis is spread from sector-to-sector.

5. In every time period (which is set to the length of a quarter) it is possible for each sector to change states once (become infectious or become susceptible). By construction, it is necessary to assume that recovery cannot occur in the same period as infection; more specifically, the frequency of the data do not enable observation of infection/recovery episodes that occur within a single time period.

6. The probability that a susceptible sector $j$ gets contaminated by an infectious sector $i$ is denoted by $p_{i j}$ and satisfies the Markov property, that is, it is independent of both the time since sector $j$ was last susceptible and $i$ was last infectious. An implication of the Markov assumption is that the length until infection of sector $j$ by infected sector $i$ therefore follows a geometric distribution.

7. The recovery probability is also assumed to be first-order Markov, that is, the probability that an infected sector recovers in a period is independent of the number of periods it has already been infected. The (sector-specific) probability that sector $i$ will recover next period is denoted by $p_{i}$; therefore the probability that sector $i$ is still infected is $\left(1-p_{i}\right)$. The mean infectious period is thus $1 / p_{i}$. The probability to still be infected after $T$ periods is therefore $\left(1-p_{i}\right)^{T}$, i.e., the probability is decreasing in $T$. Note that since we are using a discrete modeling framework, the Markov property implies that the length of the infectious period follows a geometric distribution, which is the discrete counterpart to the exponential distribution as used by Becker and Angulo (1981). ${ }^{3}$

\footnotetext{
${ }^{3}$ Note also that this assumption means that each sector's recovery probability does not depend on the other sectors and hence recovery is modeled differently than contamination (that does depend on other sectors). The benefit of this assumption is a dramatic reduction in the number of parameters
} 
8. $R_{0}$ is the basic reproduction number, a commonly used statistic in epidemiology. It is equal to the expected number of infections one infectious sector will cause in an otherwise susceptible system, that is, a system where no other sectors are infected (i.e., all other states are in state $\mathrm{S}$ ). $R_{0}$ for sector $i$ is equal to $\frac{1}{p_{i}} \sum_{j=1, j \neq i}^{N} p_{i j}$. Here it is assumed that contacts are independent, so that the probability of sector $i$ getting contaminated by sector $j$ does not change if sector $i$ is also in contact with sector $n$ in that same period. This assumption implies the following: if sector $i$ already has contaminated sector $j$ in one period, the probability that sector $i$ will also contaminate sector $k$, notated as $p_{i k}$, in that same period does not change.

Additionally to these eight assumptions, we need to introduce one additional parameter to deal with periods where no sectors are infected, which distinguishes this epidemiological model from traditional epidemiological models. In a standard epidemiological model, we might for example have a disease die out because the whole system becomes immune. In an economic setting, the concept of reaching a state of immunity is not plausible, in that it is always possible for a sector to enter a downturn or crisis via exogenous factors. It is therefore necessary for our model to allow for exogenous contamination, so that even when no sectors are infected, there is some positive probability of a given sector becoming infected in the next period. We assume that this probability is constant for all sectors and is characterized as "nature", but in principle the "nature" probability could also differ across sectors. The exact interpretation of this parameter is that it is the probability of becoming infected when no other sectors are contagious.

A visualization of the model is presented in Figure 1.

\section{[Insert Figure 1]}

that need to be estimated. It is straightforward, however, that it introduces recovery dependence along the lines of the infection dependence, where it is easier for a sector to recover if other sectors have also started to recover. Although our primary focus in this paper is the transmission of negative shocks, from a policymaker's perspective the transmission of recovery could be equally important, for example, in determining which sectors would benefit most from policy stimulus in the event of a crisis. 
The model is visualized as a Markov Chain, with the following transmission probabilities between the different states (numbered to correspond to the numbers in the figure) as derived from the above-mentioned assumptions, where S still represents the susceptible state, I represents the infectious state and $X_{i t}$ is the observed state of sector $i$ at time $t$ :

(0) $P\left(X_{i t}=S \mid X_{i t-1}=S, X_{j t-1}, \forall j \neq i\right)=1-\tilde{p}_{i t}$, where $\tilde{p}_{i t}=1-\prod_{j \in \text { infectious } t}\left(1-p_{j i}\right)$ is the probability that sector $i$ will be in contact with at least one infectious sector. (0) thus represents the probability of avoiding contamination in one period, conditional on not being contaminated in the previous period. Note that if there are more infected sectors in the network, $\tilde{p}_{i t}$ increases, indicating that it will be more likely that sector $i$ will also become infected. If no sector is currently infected, $\tilde{p}_{i t}$ is equal to the probability of being contaminated by 'nature', denoted by $n$.

(1) $P\left(X_{i t}=S \mid X_{i t-1}=I\right)=p_{i}$, which is the probability of recovering in one period, conditional on being contaminated in the previous period.

(2) $P\left(X_{i t}=I \mid X_{i t-1}=I\right)=1-p_{i}$ is the probability of not recovering (remaining infected) in one period, conditional on being infected in the previous period.

(3) $P\left(X_{i t}=I \mid X_{i t-1}=S, X_{j t-1}, \forall j \neq i\right)=\tilde{p}_{i t}$, which is the probability of being contaminated by at least one infectious sector in one period, conditional on not being contaminated in the previous period.

\section{Two-level model}

We extend the dichotomous model by introducing transition to a crisis so that there are now three different states. State $\mathrm{S}$ is still the susceptible state, as in the dichotomous model, but there are now two infectious states. In state $\mathrm{D}$, a sector has experienced just one period of net worth decline; this will now be called a downturn. In state $\mathrm{C}$, the sector is in an actual crisis, having experienced two consecutive periods of net worth decline. The introduction of the intermediate state enables a richer (and in our view, more realistic) characterization of the economy in that sectors can either improve or worsen at the same time as possibly infecting other sectors. 
We consider two versions of this specification: (i) a model where the sectors recover from a crisis directly back to the susceptible state and (ii) a model where sectors recover back to the downturn (weakened) state and from there either experience complete recovery (i.e., move back to the susceptible state) or relapse (i.e., move back to the crisis state). We will refer to these as the two-level model with immediate recovery and the two-level model with staged recovery, respectively. We discuss each model in turn.

\section{Two-level model with immediate recovery}

In the two-level model with immediate recovery, the recovery rate from state $\mathrm{D}$, that is, the probability to go from state $\mathrm{D}$ to state $\mathrm{S}$ is most likely higher than the probability of going from state $\mathrm{C}$ to $\mathrm{S}$, implying that it is probably easier to recover from a downturn than from a crisis. We initially assume that sectors are equally infectious in states $\mathrm{D}$ and $\mathrm{C}$, but this assumption can be relaxed. ${ }^{4}$ We refer to this model as the restricted two-level model; its estimates will be compared to the unrestricted two-level model with immediate recovery. Alternatively, we might instead assume that sectors are not yet infectious at all while in a downturn. This would turn the two-level model into an SEI model (Suspectible-Exposed-Infected), used by among others Bettencourt, Cintrón-Arias, Kaiser, and Castillo-Chávez (2006). We do not estimate this specific model, but it is actually a restricted version of the unrestricted two-level model that we do estimate.

Assumption 3 of the one-level model is replaced by Assumption $3^{*}$ :

$3^{*}$ A sector is susceptible as long as the growth of its net worth is positive. A sector is in state D (downturn) if it has had one period of negative net worth growth, where net worth is defined as total assets - total liabilities for that sector. A sector is in state $C$ (crisis) after two consecutive periods of negative net worth growth.

Note that with this definition, it is not possible to stay in state D for more than

\footnotetext{
${ }^{4}$ Because relaxing this assumption would lead to an additional $N *(N-1)$ parameters to be estimated, where $N$ is the total number of sectors, we retain the assumption for now. In the specification of the model (below), we also discuss what would change in the case where this assumption is relaxed.
} 
one period; a sector either recovers and goes back to being susceptible or it enters into the crisis state. It is also not possible to return from state $\mathrm{C}$ (crisis) to state D (downturn) within one single period. For an example of how the observations are labeled, consider the fourth column (labeled 'Immediate recovery') in Table 1. The other assumptions are also slightly changed to match the two-level model, these can be found in the Appendix. The transition probabilities are visualized in Figure 2 below and are stated as follows:

\section{[Insert Figure 2]}

(0) $P\left(X_{i t}=S \mid X_{i t-1}=S, X_{j t-1}, \forall j \neq i\right)=1-\tilde{p}_{i t}$, which represents the probability of avoiding contamination in one period, with $\tilde{p}_{i t}=1-\prod_{j \in D_{t}, C_{t}}^{N}\left(1-p_{j i}\right)$. As in the dichotomous model, this probability is time dependent, because it depends on the set of contaminated sectors, which varies over time. If we want to assume different infection rates in state $\mathrm{D}$ and state $\mathrm{C}$, we would define $\tilde{p}_{i t}=$ $1-\prod_{j \in D_{t}}^{N}\left(1-p_{j i}\right) \prod_{j \in C_{t}}^{N}\left(1-q_{j i}\right)$, where $p_{i j}$ is the probability that sector $i$ will infect sector $j$ if sector $i$ is in state $\mathrm{D}$ (downturn) and $q_{i j}$ is the probability that sector $i$ will infect sector $j$ if sector $i$ is in state $\mathrm{C}$ (crisis). In case no other sector is contaminated, the probability of becoming infected equals nature, $n$.

(1) $P\left(X_{i t}=D \mid X_{i t-1}=S, X_{j t-1}, \forall j \neq i\right)=\tilde{p}_{i t}$, which is the probability of being infected in one period.

(2) $P\left(X_{i t}=S \mid X_{i t-1}=D\right)=p_{i}$, which represents the probability of recovering from a downturn in one period.

(3) $P\left(X_{i t}=C \mid X_{i t-1}=D\right)=1-p_{i}$, which is the probability of not recovering from a downturn and thus entering a crisis in one period.

(4) $P\left(X_{i t}=C \mid X_{i t-1}=C\right)=1-q_{i}$, which is the probability of not recovering from (i.e., staying in) a crisis in one period.

(5) $P\left(X_{i t}=S \mid X_{i t-1}=C\right)=q_{i}$, which is the probability of completely recovering from a crisis in one period.

In the two-level model, the basic reproduction number $R_{0}$ can be defined in one of two ways. It can either be defined analogously to the dichotomous model as the 
expected number of infections sector $i$ will cause in an otherwise uncontaminated system from the moment it enters a crisis, denoted $R_{0}^{C}$, or it can be defined as the expected number of infections sector $i$ will cause in an otherwise uncontaminated system from the moment it enters a downturn (D), denoted $R_{0}^{D}$.

$$
\begin{aligned}
& R_{0}^{C}=\frac{1}{q_{i}} \sum_{j \neq i} p_{i j} \\
& R_{0}^{D}=\sum_{j \neq i} p_{i j}+\left(1-p_{i}\right) \frac{1}{q_{i}} \sum_{j \neq i} p_{i j}
\end{aligned}
$$

Relaxing the model further to allow different levels of contagiousness in states D and C, these statistics become:

$$
\begin{aligned}
& R_{0}^{C}=\frac{1}{q_{i}} \sum_{j \neq i} q_{i j} \\
& R_{0}^{D}=\sum_{j \neq i} p_{i j}+\left(1-p_{i}\right) \frac{1}{q_{i}} \sum_{j \neq i} q_{i j},
\end{aligned}
$$

where $p_{i j}$ is the probability that sector $i$ will infect sector $j$ if sector $i$ is in state D and $q_{i j}$ is the probability that sector $i$ will infect sector $j$ if sector $i$ is in state C.

Note that the idea of using a two-level model to describe the development of a disease is not new in the epidemiological literature, as it has, for example, been used to model carcinogenesis (Moolgavkar, Day, \& Stevens, 1980). Previous experimental and epidemiological evidence strongly suggested that carcinogenesis is a multistage process and Moolgavkar et al. (1980) demonstrated the added value of a two-stage model in modeling the epidemiologic features of breast cancer in females. We posit that a two-stage model might also provide a richer characterization of the development of economic crises relative to the more restrictive one-level model. A crisis is preceded by a one-period downturn, which, with adequate prevention by regulators and policymakers, may lead to recovery and avoid turning into a full-blown crisis. Thus we expect that it may be easier to recover from a downturn than from an actual crisis (defined as at least two periods of negative net worth decline). In addition, the transition from state $\mathrm{D}$ to state $\mathrm{C}$ might be interpreted as a lack of effective policy actions leading to a failure to recover from the downturn. Note that there are some key differences between the two-level model we propose and the two-level model of 
Moolgavkar et al. (1980). First of all, we use a discrete version of the two-level model; furthermore, in Moolgavkar et al. (1980), entering the second stage of carcinogenesis is more comparable to a new 'infection', rather than a failure to recover from the first.

\section{Two-level model with staged recovery}

An alternative two-level model is proposed in this section: the two-level model with staged recovery. The Markov chain of this model differs from the previous one in the sense that when a sector recovers from a crisis, it is not fully recovered; rather it moves back to the downturn stage (referred to as a 'fragile period'). From this fragile period, the sector can either (a) fully recover and thus enter the susceptible state, or (b) fail to recover and re-enter (slide back into) the crisis state again, that is, it experiences a relapse. This variation of the model is considered as a plausible alternative representation of the economy where recovery from a crisis is neither guaranteed nor smoothly occurring. To provide some motivation for also considering the two-level model with staged recovery, the Data section will demonstrate that relapses occur with fairly high frequency.

The model with staged recovery is very similar to the two-level model with immediate recovery, which can be seen by comparing the visualization of this model (Figure 3) to the visualization of the two-level model with immediate recovery in Figure 2. Modeling the staged recovery Markov chain instead of the one associated with immediate recovery requires very few adaptations. The main change is in the classification of the data episodes. Instead of classifying the period after a sector exits a crisis as being in the susceptible state, we instead classify it as in a downturn again. ${ }^{5}$

\section{[Insert Figure 3]}

Due to reclassification of the data, the computation of $R_{0}^{D}$, the basic reproduction number, also changes. $R_{0}^{C}$, the expected number of infections sector $i$ will cause in an

\footnotetext{
${ }^{5}$ Note that given our definition of a crisis, i.e., two consecutive periods of negative net worth growth, it is still not possible for a sector to be in a downturn for more than one period. This requirement is not restrictive and is easily modified by introducing a more negative (lower) threshold to define a crisis, e.g., two consecutive periods of a more than $2 \%$ contraction. This modified specification allowing for protracted downturn episodes would introduce an additional set of transition probabilities (i.e., the probability of being in a downturn conditional on already being in a downturn).
} 
otherwise uncontaminated system from the moment it enters a crisis, does not change and is still computed as in Equation 1. The expected number of times a sector will enter a crisis from the moment it enters a downturn is given by $\frac{1-p_{i}}{p_{i}}$ (the expected value of a geometric distribution starting at zero). Every period it is in a downturn, it is expected to infect $\sum_{j \neq i} p_{i j}$ other sectors (assuming no one is infected yet) and will on average be in a downturn for $\left(\frac{1}{p_{i}}\right)$ periods. Once it enters a crisis, it will on average infect $\frac{1}{q_{i}} \sum_{j \neq i} p_{i j}$ other sectors (assuming no other sectors are infected yet). Thus, this gives us

$$
R_{0}^{D}=\frac{1-p_{i}}{p_{i}} \frac{1}{q_{i}} \sum_{j \neq i} p_{i j}+\frac{1}{p_{i}} \sum_{j \neq i} p_{i j} .
$$

\section{Comparisons with other Markov-based econometric models}

Due to their specification in terms of Markov chains, the epidemiological models bear some resemblance to a number of other models that are more commonly used in economics. One of the most common methods for describing the transmission of shocks is via impulse response functions derived from a vector autoregression (VAR) framework. The standard VAR does not admit states, per se, but rather characterizes the sectors' net worth as evolving according to a Markov process. The connections across sectors are captured through the covariance matrix of shocks to each sector, as well as via the transition matrix itself. These models typically focus on steady state dynamics and as such fall short in characterizing rapid changes such as those that are typically seen in a crisis environment.

One way of trying to capture the lagged propagation of return spillovers in the financial system has been through the use of Principal Components Analysis to identify unobserved states combined with a Generalized Autoregressive Conditional Heteroskedasticity (GARCH) model to design a Granger-causality measure of connectedness, as proposed by Billio, Getmansky, Lo, and Pelizzon (2012). Their Degree of Granger Causality (DGC) measures the fraction of statistically significant Grangercausality relationships among $N(N-1)$ pairs of $N$ financial institutions but could similarly be applied in our sector context. The DGC bears some resemblance to our basic reproduction number (the $R_{0}$ statistic), which captures the expected number of contagions originating from one sector in an otherwise uncontaminated system. However, there is an essential difference in that with the DGC measure, only the effects 
of a change in sector $i$ 's net worth in period $t$ on the other sectors in the following period are known. In contrast, the benefit of the epidemiological approach is that the basic reproduction number identifies the expected number of sectors that will similarly enter a downturn or crisis state via contamination from sector $i$, not just over the next period but during the whole period that sector $i$ is expected to be in a downturn/crisis.

As noted in the introduction, due to their specification in terms of Markov chains, the proposed models are somewhat related to Markov switching models (Hamilton, 1989). To demonstrate the similarities and differences, suppose one would want to model the net worth growth $y$ of sector $i$ using a simple two-state Markov switching model:

$$
y_{i t}=\mu_{i, x_{i t}}+\varepsilon_{i t}
$$

with $x_{i t}=0$ or 1 denoting the realized value of the state $X_{i t}$. For each sector $i$, the state $X_{i t}$ follows a sector-specific first-order Markov process with transition probabilities $P\left(X_{i t}=0 \mid X_{i, t-1}=0\right)=p_{i}$ and $P\left(X_{i t}=1 \mid X_{i, t-1}=1\right)=q_{i}$. A very important difference between the epidemiological models and the Markov switching models is that in Markov switching models, the states $X_{i t}$ are unobserved and are endogenously determined according to whether the estimated probabilities are above or below a chosen threshold. In the epidemiological literature, the states are defined exogenously and hence are a priori observed — these are then subsequently modeled based on a Markov chain.

Furthermore, in the standard Markov switching model, transition probabilities are assumed to be constant. In our epidemiological model, the transition probabilities of entering a downturn $\left(P\left(X_{i t}=D \mid X_{i t-1}=S, X_{j t-1}, \forall j \neq i\right)=\tilde{p}_{i t}\right)$ are timevarying and in fact depend on which other sectors are infectious at that moment in time, which is essential for capturing the idea of contagion. Advances have been made in Markov switching models by making the transition probabilities depend on explanatory variables (Diebold, Lee, \& Weinbach, 1994), but not yet in the direction of estimating a system of Markov switching models where transition probabilities depend on values of other unobserved state variables. Among others Guidolin and Timmermann (2007) did consider the estimation of a multivariate regime switching model, but each regime is still the realization of a first-order Markov chain with 
constant transition probabilities. Integrating the epidemiological framework into the family of regime switching models would be an interesting addition to the current literature, as it would allow us to endogenize states; this remains, however, a topic for future research.

Our model is also related to the discussion in the literature on methods for dating business cycles (Hamilton, 2003; Harding \& Pagan, 2003a, 2003b) that compares Markov switching models with a nonparametric algorithm providing a good approximation to the chronology determined by the National Bureau of Economic Research (NBER). ${ }^{6}$ At some level, the debate rests on an important philosophical distinction in that the Markov switching model attempts to model whether a real event did or did not occur and hence is event-specific while the nonparametric approach is a simple rule that can be applied irrespective of the data or purpose. Proponents of the latter approach (e.g., Harding and Pagan, 2003a) argue that such an approach is preferable on grounds of transparency, robustness, simplicity, and replicability. Our epidemiological models in some ways blend these two approaches. On one hand, like the nonparametric literature we use "observed states", i.e., we define downturn and crisis states based on a number of periods of negative growth - these can be interpreted as akin to a nonparametric window of width one or two periods (that could be widened if desired). ${ }^{7}$ But on the other hand, similar to Markov switching models, we model the transitions between states as Markov chains. A further criticism of the nonparametric approach (Hamilton, 2003) is that it cannot be easily applied in a system (vector) context. While our epidemiological models also estimate each sector separately, the cross-sector transition probabilities admit some limited system interaction.

\section{Data and Descriptive Statistics}

\section{The Fourteen Sectors}

The publicly available Financial Accounts of the United States - Z.1 ("Flow of Funds") data, comprising the entire set of financial accounts of the United States economy,

\footnotetext{
${ }^{6}$ We thank Dick van Dijk for calling attention to this debate in the context of our models.

${ }^{7}$ Harding and Pagan (2003a) note that the algorithm (Bry \& Boschan, 1971) included some additional strictures to ensure cycles had minimum durations - the epidemiological model could be similarly adapted to include such constraints although we refrain from doing so since our focus is on shorter-term transitions rather than the identification of economic cycles.
} 
are used for analysis. ${ }^{8}$ These data were reported annually until 1952 and quarterly thereafter. Our sample period runs from the first quarter of 1952 to the fourth quarter of 2015, resulting in 256 quarterly observations for each sector we consider. The data are organized into two subcategories, (1) Assets \& Liabilities, and (2) Flows. The accounts are again further subdivided into different sectors of the United States Economy as shown in Figure 4. Due to data availability, the following fourteen sectors (shown in grey in Figure 4) are used in the model: Household and Nonprofit Organisations (HH\&NP), Noncorporate Nonfinancial Business (NNB), Corporate Nonfinancial Business (CNB), State and Local Governments (S\&LG), Federal Government (FG), Rest of the world (W), Monetary Authority (MA), Life Insurance Companies and Property-Causality Insurance Companies (IC), Private Depository Institutions and Money Market Mutual Funds (PDI\&MMMF), Private and public pension funds (PF), Financial Companies (FinC), Government Sponsored Enterprises (GSE), Security Brokers and Dealers (SB\&D) and Other (Other). The components of Other are also shown in Figure 4.

\section{[Insert Figure 4]}

The difference between the assets and liabilities of each sector (net assets) is used to calculate the net growth for each sector (i.e., the change in net assets over two consecutive periods); it is this variable that is used to determine the state the sector is in (susceptible, downturn, crisis) as described in the model section. For a compact visualization of these net worth growth series, consider Figure 5, where white areas indicate very low levels and black areas indicate very high levels of net worth growth, ${ }^{9}$ together with a graph of how the median net worth growth over all sectors evolves over time (lower graph) and a box plot showing the median and range of net worth growth over the sample period for each individual sector (right box), based on the visualization method of Peng (2008). The median over all sectors supports the use of net worth growth as a proxy for dating downturns and crises, as its most recent

\footnotetext{
${ }^{8}$ These data are available from website of the Board of Governors of the Federal Reserve System (2016) (Financial Accounts of the United States, Z1, "Flow of Funds", http: //www.federalreserve .gov/releases/z1/)

${ }^{9}$ This visualization is constructed by applying a smoothing spline with 25 degrees of freedom to each individual time series and discretizing the range of net worth growth into 10 equal-sized buckets.
} 
troughs coincide with the latest National Bureau of Economic Research (NBER) recessions, that have been highlighted in the same panel.

\section{[Insert Figure 5]}

The subdivision of the economy into fourteen sectors allows for quite a detailed analysis of the economy. In 2015Q3 for example, the household and nonprofit organizations (HH\&NP) sector is the largest sector with $35 \%$ of the total assets, followed by the corporate nonfinancial business (CNB) sector with a share of $14 \%$. The share of the security brokers and dealers sector in total assets is only $1 \%$.

\section{Summary statistics on dichotomous and two-level epidemiological models}

Descriptive statistics for the two-level model with immediate recovery are shown in Table 2. Descriptive statistics related to the dichotomous model for the fourteen sectors are not given in a separate table, as they are in fact overlapping with the summary statistics of the two-level model with immediate recovery that will be discussed below. This is the case because in the dichotomous model, the downturn and crisis states are simply aggregated into a single state ('I' in the dichotomous model). ${ }^{10}$

\section{[Insert Table 2]}

The empirical probabilities of recovering from a downturn (column 4) are all above 0.5, meaning that for all sectors, recovery is more likely than moving into a crisis. Should a sector fall into a crisis, however, the outlook changes. The empirical probabilities of recovering from a crisis (column 5) for the NNB, CNB and PDI\&MMMF sectors are all relatively low, below 0.5 , meaning there is a greater probability of staying in a crisis than recovering from it; an additional three sectors have empirical recovery probabilities equal to 0.5 . Note that from the Markov property the average length of

\footnotetext{
${ }^{10}$ Recall the differences in how episodes are labeled that result from the different definitions, illustrated in Table 1. It is evident that the average time that a sector is infectious (not susceptible) increases in the staged recovery specification relative to both the immediate recovery model and the dichotomous model. In the two-level staged recovery model, a single period with positive net worth growth directly after a crisis period is labeled as a downturn period, not as a susceptible period. Thus, if a relapse occurs, this becomes a single long period where the sector alternates between crisis and downturn periods, instead of multiple short crises/downturn periods.
} 
time in an infected state is $\frac{1}{p_{i}}$ so that for the six sectors with a recovery probability equal to or below 0.5 , the mean recovery time from a crisis is greater than two periods. All other sectors have a greater probability of recovering than staying in crisis. Because a sector can contaminate more than one sector each period, additional summary statistics on the number of contacts (contaminations) between each sector are given in Table $\mathrm{C} 1$ in the Appendix.

It is interesting to compare these recovery probabilities with transition probabilities found via Markov switching models for the United States as a whole, even though as described above these models have some important differences. Hamilton (1989) estimated a recovery probability of 0.245 , which is remarkably lower than the recovery probabilities our epidemiological model specification suggests. This may be because transitions in individual sectors are a lot more dynamic than for the United States as a whole, which would result in higher recovery probabilities and higher probabilities to enter a crisis as well. We explore this possibility further later in the paper.

One way to characterize the dynamics of crises is via an epidemic curve showing the total number of sectors in crisis at each time period. The epidemic curve corresponding to our alternative definition where crises are preceded by downturns is given in Figure 6 below. We can see that at almost every point in time, at least one sector seems to be in a downturn/crisis and on average 3.4 sectors are in either a downturn or a crisis in any given period. For comparison purposes, bars corresponding to the NBER recessions are included in the figure. While it is apparent that some of the periods with multiple sectors in crisis often correspond to NBER recession periods, in particular the 2007-08 crisis and the early 2000s recession, in other cases it is less apparent. It is notable that this figure is consistent with the interpretation that the most widespread crisis in this sample was during the financial crisis of 2007-08, where at its peak 11 different sectors were contaminated.

\section{[Insert Figure 6]}

Figure 7 shows a disaggregated version of the epidemic curve by sector, where in addition to seeing which sectors are in crisis in each period, it is also possible to see the length of time a sector stays in crisis. These are the data used as inputs for the two-level model with immediate recovery. From this figure, we see that some sectors 
experience considerably fewer downturns/crises than others and hence it is important to recognize that the definition of a downturn or crisis may differ in each sector. While the focus of this paper is on the transmission of downturns or crises across sectors once they are detected and not the labeling method itself, to check for the possibility that the results are influenced by the definitions/labeling, the robustness section explores alternative methods for labeling of the downturns and crises. This includes an approach that normalizes the number of downturns to be the same (i.e., a constant proportion of quarters) across sectors. Additionally, the robustness section analyzes whether the results are similar when the underlying net worth growth series is deseasonalized before labeling the downturns/crises.

\section{[Insert Figure 7]}

As indicated in the section discussing the two-level model with staged recovery, relapses occur with very large frequencies, supporting that the use of the two-level model with staged recovery might indeed be of added value. Results supporting this claim are given in Table 3. This Table depicts the number of times a sector immediately enters a downturn, after it just recovered from a crisis divided by the total number of times it recovers from a crisis.

\section{[Insert Table 3]}

\section{Results}

\section{Estimation}

The parameters were estimated via maximum likelihood. For the dichotomous model, this includes the estimation of the contact probabilities $p_{i j}$ and the recovery probabilities $p_{i}$, for $i=1, \ldots, N$ and $j=1, \ldots, N, j \neq i$, as well as the nature parameter, $n$. For the restricted two-level models, this involves estimating the contact probabilities $p_{i j}$, the recovery probabilities from a downturn $p_{i}$, the recovery probabilities from a crisis $q_{i}$, and nature, $i=1, \ldots, N$ and $j=1, \ldots, N, j \neq i$. For the unrestricted twolevel model, we also have to estimate $q_{i j}, i=1, \ldots, N, j=1, \ldots N, j \neq i$, the contact probabilities once in a crisis, while $p_{i j}$ becomes the contact probability in a downturn. 
The likelihood of sector $i$ at time $t$ in the dichotomous model is built up from these probabilities and is given by:

$$
\mathscr{L}_{i, t}= \begin{cases}1-\tilde{p}_{i t} & \text { if } X_{i t}=S \text { and } X_{i t-1}=S \\ \tilde{p}_{i t} & \text { if } X_{i t}=I \text { and } X_{i t-1}=S \\ p_{i} & \text { if } X_{i t}=S \text { and } X_{i t-1}=I \\ 1-p_{i} & \text { if } X_{i t}=I \text { and } X_{i t-1}=I .\end{cases}
$$

where $\tilde{p}_{i t}=1-\prod_{j \in I_{t}}\left(1-p_{j i}\right)$. We aggregate the individual likelihoods by multiplying the sector-and-time specific likelihoods for all sectors $i=1, \ldots, N$ and all observations in time $t=1, \ldots, T$ to obtain the full likelihood. The equivalent for the two-level model with immediate recovery is given by Equation (7). In this equation, $\tilde{p}_{i t}=$ $1-\prod_{j \in D_{t}, C_{t}}\left(1-p_{j i}\right)$.

$$
\mathscr{L}_{i, t}= \begin{cases}1-\tilde{p}_{i t} & \text { if } X_{i t}=S \text { and } X_{i t-1}=S \\ \tilde{p}_{i t} & \text { if } X_{i t}=D \text { and } X_{i t-1}=S \\ p_{i} & \text { if } X_{i t}=S \text { and } X_{i t-1}=D \\ 1-p_{i} & \text { if } X_{i t}=C \text { and } X_{i t-1}=D \\ q_{i} & \text { if } X_{i t}=S \text { and } X_{i t-1}=C \\ 1-q_{i} & \text { if } X_{i t}=C \text { and } X_{i t-1}=C\end{cases}
$$

To estimate the dichotomous model, 14 recovery probabilities, $14 \times 13=182$ contamination probabilities and one nature parameter have to be estimated; we discuss the results of this model only briefly. In order to estimate the restricted two-level model, we need to estimate (for 14 sectors) 14 recovery probabilities $p_{j}, 14$ recovery parameters $q_{j}, 14 \times 13=182$ contamination probabilities $p_{i j}$ and 1 parameter that captures the probability of being infected by nature, which in total sums up to 211 transition and contamination probabilities. For the unrestricted model, this becomes $14+14+(2 \times 14 \times 13)+1=393$ parameters. Although the number of parameters may seem large at first, the model remains tractable since multiple groups of parameters can be estimated independently of each other. This can be seen from the first and second order derivatives of the likelihood function that have been derived in Ap- 
pendix B. For example, every recovery probability $p_{i}, i=1, \ldots, N$ can be seen to be independent of all other parameters, as their derivatives only depend on $p_{i}$ itself (see Equation B.2). The same holds true for $q_{i}$.

The derivatives of the $\log$-likelihood function $l_{i t}$ with respect to $p_{j i}$ (Equations B.4B.6) for the restricted two-level models only depend on the parameters $p_{k i}, k \neq i$, implying that $p_{j i}$ can be estimated independently of most of the parameters other than the set of probabilities corresponding to sector $i$ being infected, $\left\{p_{k i}\right\}_{k=1, k \neq i}^{N}$. In both the dichotomous model and the restricted two-level models, these sets of dependent parameters contain at most $N-1=13$ elements. In the unrestricted two-level model with immediate recovery, this set of dependent parameters contains at most 26 elements, because the probability $\tilde{p}_{i t}$ that enters the likelihood function is now built up from the set of parameters $\left\{p_{j i}, q_{j i}\right\}_{j=1, j \neq i}^{N}$.

Because $R_{0}$ is a nonlinear transformation of the parameters, standard errors for the $R_{0}$ statistics have been computed using the delta method. However, these standard errors are not available for some parameters of some models, as for some model specifications there are not enough observations for each contamination probability $p_{i j}$ (and $q_{i j}$ as well in the unrestricted specification). ${ }^{11}$ This is because in the observed data, there are some cases where a specific sector (e.g., sector 'i') is only once (or never) in a crisis or downturn at the moment another sector (e.g., sector ' $j$ ') could have gotten infected. In this case, there is no variation in the estimated probability by which an estimated standard error could be obtained. This is only the case for selected parameters in the unrestricted two-level model with immediate recovery and in the robustness analysis of the restricted two-level model with staged recovery where the sample is split in two. Standard errors for the recovery probabilities are for example always available, but this is not the case for all transmission probabilities.

\section{Dichotomous model}

The estimates of $R_{0}$ for the dichotomous model are given in Table $4 .{ }^{12}$ The estimated log-likelihood is equal to -1788.6, but cannot be compared directly with the two-level models, as it does not describe the same data (i.e., in the dichotomous specification,

\footnotetext{
${ }^{11}$ The notes to the tables identify where this situation arises.

${ }^{12}$ All estimated parameters can be found in the Supplementary Online Appendix, which includes the estimations of the transmission probabilities and recovery probabilities.
} 
there is no such thing as a downturn state - they are always labeled as a crisis). The results suggest that the least infectious sector is the Monetary Authority (MA), and the most infectious sectors are Corporate Nonfinancial Business (CNB), Insurance Companies (IC) and Private Depository Institutions and Money Market Mutual Funds (PDI\&MMMF). If PDI\&MMMF enters state ' $\mathrm{I}$ ' and thus becomes infectious, it on average infects 2.4 other sectors in an otherwise uncontaminated system.

[Insert Table 4]

\section{Restricted two-level model with immediate recovery}

We first estimate a restricted version of the two-level model in which the sectors are assumed to have equal contamination probabilities in both state D (downturn) and state $\mathrm{C}$ (actual crisis). This is done to limit the number of parameters and to simplify the computations of the likelihood function and its derivatives. The estimated contamination probabilities are given in Table 5, together with the estimated recovery probabilities from respectively state $\mathrm{D}\left(p_{i}\right)$ and state $\mathrm{C}\left(q_{i}\right)$. As can be seen, the recovery probabilities coincide with those given in columns 4 and 5 in Table $2 .{ }^{13}$ Note the substantial variation in the contamination probabilities across sectors. For example, PDI\&MMMF and MA are on average more likely to become infected than NNB; in the latter sector, contamination probabilities are mostly below $10 \%$ (as seen by the column labeled "NNB" in the table), meaning that the NNB sector is reasonably insulated from infection. The estimated contamination probabilities also provide some insight into the potential sequencing of contamination should an economy-wide crisis develop. For example, there is a more than $23 \%$ probability that PDI\&MMMF will become contaminated if IC has a downturn or crisis, while a crisis in PDI\&MMMF has little likelihood of spreading to the HH\&NP, NNB, CNB, or SL\&G sectors. As described in the model description, an additional parameter has been introduced that captures the probability of entering a crisis exogenously (i.e., when there is no other sector in a downturn or crisis to cause an infection), the 'nature' parameter, $n$. This parameter is estimated to be $0.27(0.04)$. The interpretation of this parameter is that

\footnotetext{
${ }^{13}$ The reason for this is that the recovery parameters can be estimated independently from the transmission probabilities in all models under consideration. This can be seen in Appendix B, where the first-order derivatives of the log-likelihood functions with respect to the recovery probabilities are shown to be independent of the values of the transmission probabilities.
} 
when no sectors are currently in a downturn or a crisis, the probability that some sector will enter a downturn in the next period is on average $27 \%$. The magnitude of this parameter is intuitively reasonable given that the average number of sectors in a downturn in any given quarter is 3.4 (see Figure 6).

\section{[Insert Table 5]}

In Table 6, we report the basic reproduction numbers $\left(R_{0}\right)$, calculated using the estimated parameters from Table 5. $R_{0}^{D}$ of sector $j$ represents the expected number of contaminations in an otherwise uncontaminated system originating from sector $j$ from the moment that sector enters a downturn. $R_{0}^{C}$ similarly gives the expected number of contaminations in an otherwise uncontaminated system from the moment the sector enters a crisis. The most contagious sector is PDI\&MMMF, private depository institutions and money market mutual funds; on average 3.6 subsequent contaminations will originate from this sector once it enters a downturn and 5.2 once it enters a crisis. Also very contagious are the NNB (noncorporate nonfinancial businesses) and CNB (corporate nonfinancial businesses) sectors. The least contagious sector is MA, the monetary authority sector. The two different $R_{0}$ statistics suggest that the NNB and PDI\&MMMF sectors are especially contagious in a crisis, as the expected number of contaminations originating from a sector once in a crisis, $R_{0}^{C}$, is a lot higher than $R_{0}^{D}$, the expected number of infections originating from a sector once in a downturn.

\section{[Insert Table 6]}

An alternative approach to quantify the contagiousness of a single sector is to look at the probability of infecting no more than $0,1,2,3, \ldots$ other sectors in a single period in an otherwise uncontaminated system. This cumulative probability is visualized in Figure 8. The difference between this statistic and the basic reproduction number, $R_{0}$, is that this statistic does not take into account how long a sector is expected to stay infectious. From this figure we observe that the monetary authority has the highest probability of not infecting any other sector; the probability that it infects no sector at all in a given period is almost 0.4 , while for the most infectious sector, PDI\&MMMF, this is around 0.25. Similarly, the probability that the MA sector infects no more than one other sector is nearly 0.8 while for the PDI\&MMMF sector 
it is only 0.6. For all sectors, the probability of infecting no more than 4 additional sectors is near 100 percent (i.e., the cumulative probability reaches 1.0 when $x=4$ ).

\section{[Insert Figure 8]}

Figure 9 graphs the probability that each sector will become infected at a specific moment in time. Here becoming infected refers to the probability of entering state D (the downturn state). Entering a full crisis (state C) depends both on this initial probability and the ability of the sector to recover. As can be seen, although the NNB and CNB sectors are quite infectious (Table 6), their average probabilities of entering a downturn are relatively low; in fact, they are on average the lowest of all sectors. This is not the case for the PDI\&MMMF sector, which is both very infectious (highest $R_{0}^{D}$ and $R_{0}^{C}$, the expected number of contaminations originating from the specific sector once entering a downturn or crisis, respectively) and generally has a high probability of entering a crisis. Also interesting are the very high probabilities that both the Insurance Companies (IC) and the Monetary Authority (MA) have of entering a downturn, especially at the beginning of 2008. Despite these similarities, as shown above, the MA sector will on average infect few other sectors and has very high recovery probabilities from both a downturn and a crisis (above 0.8 in both cases).

\section{[Insert Figure 9]}

It is possible to compare the infection probabilities $\left(\tilde{p}_{i t}\right)$ in Figure 9 with the transition probability found by Hamilton (1989) for the United States as a whole in a Markov switching model, which was equal to approximately 0.1 . The restricted two-level epidemiological model with immediate recovery finds average transition probabilities varying between 0.11 for $\mathrm{CNB}$ and $\mathrm{NNB}$ to 0.41 for MA. The large variation in estimated transition probabilities emphasizes why it is important to consider the transmission of crises across individual sectors, as sectors allow us to capture the dynamic nature in a way that is not possible when considering the aggregate United States. Note that CNB accounted for $14 \%$ of the total assets in 2015Q3, while NNB and MA were $6 \%$ and $2 \%$, respectively. In Figure 10, a weighted average of the individual $p_{i t}$ 's is visualized, with the share of total assets used as time-varying weights. 
The average of this time series over the full time period is equal to 0.185 . The range that the infection probabilities in Figures 9 and 10 attain underscores the importance of using a model that allows for time-varying transition probabilities, such as the epidemiological model employed in this paper.

\section{[Insert Figure 10]}

\section{Unrestricted two-level model with immediate recovery}

In this subsection, the unrestricted two-level model and its estimation results are discussed. In this model, not only are there different recovery probabilities in the different states, but also different levels of contagiousness, giving a total of 393 parameters. ${ }^{14}$ The computed estimates for $R_{0}$ are given in Table 7 . The estimates of all parameters are given in the Appendix, Table D2. Note that there is only a little difference between the $R_{0}$ estimates of the unrestricted two-level model and those of the restricted model. Conclusions regarding the contagiousness of each sector do not change. This underscores the robustness of the models with respect to the assumptions on the contamination probabilities. Some observations on the probabilities $q_{i j}$ and $p_{i j}$ are the following:

\section{[Insert Table 7]}

(1) The PDI\&MMMF sector was already seen to be very contagious in the restricted two-level model, especially towards the monetary authority sector, with an infection probability of 0.287 . This finding is even more apparent in the full two-level model, where we observe that when PDI\&MMMF is in a crisis $(C)$, the probability that it will infect MA is equal to 0.380. (2) For most sectors, we can see that the probability to infect in a downturn is lower than the probability to infect in a crisis. Although the monetary authority is least infectious in general, it has the largest increase in contamination probabilities moving from a downturn into a crisis. On the other hand, the insurance companies (IC) have higher contamination probabilities in a downturn

\footnotetext{
${ }^{14}$ This may at first glance seem like a large number of parameters to estimate, but relative to the size of the dataset (a panel with 14 sectors and 255 observations per sector, that is, $14 \times 255=$ 3570 observations), it is manageable, although in some cases (as noted) we are unable to compute standard errors with respect to the expected number of contaminations for some sectors, $R_{0}$.
} 
than in a crisis. Contamination probabilities alone, however, do not say anything about a sector's overall infectiousness, because they do not take into account how long a sector is in each state. These observations are illustrated in Figure 11, where the cumulative probability of infecting no more than $0,1,2,3, \ldots$ other sectors in a single period in an otherwise uncontaminated system is visualized. Here we see that when the MA sector is in a downturn state, the probability of infecting no other sectors is around 0.39 , and the probability of infecting at most one other state jumps to 0.79 . In contrast, when the MA sector is in a crisis state, these probabilities decline to 0.27 and 0.64 , respectively.

\section{[Insert Figure 11]}

\section{Comparison of unrestricted and restricted two-level model with immediate recovery}

We can compare the two models using the likelihood ratio test, as the restricted twolevel model assumes $p_{i j}=q_{i j}$, while the unrestricted two-level model does not and hence the unrestricted model nests the restricted one. The null hypothesis is then $H_{0}: p_{i j}=q_{i j} \forall i, j$. This gives the following test statistic:

$L R=2 \times[\log$-likelihood full model $-\log$-likelihood restricted model $] \sim \chi^{2}(182)$.

The likelihood ratio test statistic is thus equal to $2 \times(-1755.215+1778.294)=46.16$, substantially smaller than the $95 \%$ critical value of 214.5 . Therefore, we do not reject the null hypothesis, indicating that the unrestricted two-level model does not represent a significant improvement on the restricted two-level model.

\section{Two-level model with staged recovery}

In this section, the estimation results for the two-level model with staged recovery are discussed. Because (as noted in the previous section) the unrestricted model does not significantly alter the estimates from the restricted two-level model with immediate recovery, the estimation results for all parameters in this model are relegated to Table D3 in the Appendix. The log-likelihood value of the model is -1786.81, but is not directly comparable to the log-likelihood values from the other models, as they are not nested (the data have been reclassified). The expected numbers of 
contaminations, $R_{0}$, computed based on the parameter estimates from this model, are shown in Table 8. It appears that the assumption of staged recovery leads to much higher $R_{0}$ estimates. This is primarily because the average time a sector is in either a downturn or crisis has increased. Despite this, however, the main conclusions are unchanged, regarding the large infectiousness of the PDI\&MMMF, NNB and CNB sectors, since these sectors are still most infectious. The expected numbers of contaminations in a downturn, $R_{0}^{D}$, also suggest large infectiousness for the Insurance Companies, but this is not as prominent in $R_{0}^{C}$ (the infectiousness once entering a crisis). Again, MA is least infectious, but FG (federal government) also has low infectiousness, especially according to the expected number of contaminations once in a crisis, $R_{0}^{C}$. The estimated standard errors of the $R_{0}$ 's of the Monetary Authority sector are noticeably smaller than for the other sectors. Furthermore, although both the model with immediate recovery and this staged recovery model are computed using Equation 1, the standard errors for $R_{0}^{C}$ are smaller in the staged recovery model; compare Table 8 to Table 6 . This suggests that the model that allows for staged recovery may provide a closer representation to the underlying data.

[Insert Table 8]

\section{Aggregation of sectors}

As described in Section II, it is possible to aggregate sectors if there are reasons to believe they are homogeneous. However, although parameters for some sectors may be similar, homogeneity requires not only that the recovery probabilities are more or less equal, but also the probabilities of infecting sectors and being infected by sectors. For the dichotomous model, this would already impose 14 parameter restrictions on the model when wanting to aggregate 2 sectors. For example, in the two-level model with immediate recovery, HH\&NP and GSE have similar recovery probabilities, but the probability that GSE gets infected by PDI\&MMMF has an estimated probability of 0.101 ; for the HH\&NP this is 0.015 . Furthermore, the nature of these two sectors does not offer a motivation for their homogeneity. Sensible candidates for aggregation would be CNB and NNB, two sectors that in fact have very similar parameters in the two-level model with immediate recovery. However, we see that $R_{0}^{C}$ varies as much as 0.5 between the two sectors. 


\section{Robustness}

Robustness of our results to a variety of alternative model specifications and assumptions is explored in the Appendix. In particular robustness over time, robustness to the crisis definition and robustness with respect to possible seasonality in the underlying data are considered. We find that the contagiousness of most sectors remains fairly constant over time, with the exception of the Private Depository Institutions and Money Market Mutual Funds (PDI\&MMMF) and Insurance Companies (IC) sectors. The former exhibits a strong decrease in contagiousness over time, the latter a strong increase.

When considering the robustness to the crisis definition, one alternative definition we explore is one where every sector is in a downturn/crisis exactly $25 \%$ of the time, that is, by choosing a sector-specific threshold and crisis definition. Although this leads to a decrease in the contagiousness of PDI\&MMMF (as a result of it having relatively fewer crises and the other sectors having relatively more under the alternative definition), it is still one of the most contagious sectors of the system. Under this alternative definition, the noncorporate nonfinancial business sector is the most infectious, while it was the second most infectious sector under our original definition.

To explore the possibility that some of the episodes that are labeled as downturn$\mathrm{s} /$ crises are actually related to regular seasonal fluctuations and should not cause alarm, the analyses are repeated using data that have been deseasonalized by first regressing net worth growth on a set of monthly dummies and using the residuals from this regression to identify episodes of negative net worth growth. The robustness section demonstrates that the general conclusions remain the same, that is, PDI\&MMMF, NNB and CNB remain the most infectious sectors and MA is the least infectious, even after monthly seasonal fluctuations are taken into account.

\section{Conclusion}

This paper has considered the estimation of multiple epidemiological models to analyze the spread of crises across macroeconomic sectors in the United States. The different parameters and statistics described in the previous section clearly distinguish between sectors that are either very contagious (e.g. PDI\&MMMF, NNB, CNB, IC) 
or less contagious (MA, GSE, FG), as can be seen from their either quite high or low $R_{0}$. We can also differentiate the ease with which various sectors are infected. Least likely to get infected are NNB and CNB. More likely to get infected are MA, IC and PDI\&MMMF. The very high likelihood that PDI\&MMMF will enter a crisis is especially worrisome, considering that is also the most contagious sector. The model has been shown to be robust to a relaxation of the assumptions on the contamination probabilities, as conclusions remain the same when considering the unrestricted two-level model and the restricted two-level model. The conclusions derived from the two-level model with staged recovery are also very much comparable to those of the other models. This alternative characterization sketches a more worrisome picture, however, with higher infectiousness in general, caused by the high probabilities of a relapse - suggesting that complete recovery is not so easy to achieve. The results from estimating the restricted two-level model with immediate recovery on two subsamples also suggest stability over time for the $R_{0}$ of most sectors. Two sectors appear to have changed over time; PDI\&MMMF has become less infectiousness over time and IC has become more infectiousness over time.

The models developed in this paper have demonstrated that epidemiological models can generate useful insights regarding the interconnectedness between broad macroeconomic sectors of the United States and the likelihood of a spillover occurring if one sector experiences an economic contraction. Such findings may be helpful to policymakers in determining the allocation of limited resources in times of crisis. An immediate extension of this framework would be to apply these models to analyze cross-country contagion and spillover, by considering decreases in gross domestic product of countries instead of decreases in the net worth of sectors. Another extension would be to determine the differences between sectors that give rise to our results. For example, a possible explanation of why some sectors are more infectious than others might be due to the leverage ratios (total assets divided by total liabilities) of the respective sectors over time. For example, PDI+MMMF and IC's leverage ratios show a strong upward trend around 2009, while for the majority of other sectors this trend was downward. We leave such investigations for future research. 


\section{References}

Becker, N., \& Angulo, J. (1981). "On Estimating the Contagiousness of a Disease Transmitted from Person to Person". Mathematical Biosciences, 54(1-2), 137154.

Bettencourt, L. M., Cintrón-Arias, A., Kaiser, D. I., \& Castillo-Chávez, C. (2006). "The Power of a Good Idea: Quantitative Modeling of the Spread of Ideas from Epidemiological Models". Physica A: Statistical Mechanics and its Applications, $364,513-536$.

Billio, M., Getmansky, M., Lo, A. W., \& Pelizzon, L. (2012). "Econometric Measures of Connectedness and Systemic Risk in the Finance and Insurance Sectors". Journal of Financial Economics, 104 (3), 535-559.

Board of Governors of the Federal Reserve System. (2016, March). Z1-financial Accounts of the US Economy. Available at http://www. federalreserve.gov/ releases/z1/, accessed on March 5, 2016.

Boers, F. (1997). "'No Pain, No Gain' in a Free Market Rhetoric: A Test for Cognitive Semantics?". Metaphor and Symbol, 12(4), 231-241.

Bry, G., \& Boschan, C. (1971). "Standard Business Cycle Analysis of Economic Time Series". In Cyclical Analysis of Time Series: Selected Procedures and Computer Programs (pp. 64-150). New York: National Bureau of Economic Research.

Demiris, N., Kypraios, T., \& Smith, V. L. (2014). "On the Epidemic of Financial Crises". Journal of the Royal Statistical Society: Series A (Statistics in Society), $177(3), 697-723$.

Diebold, F. X., Lee, J.-H., \& Weinbach, G. C. (1994). "Regime Switching with Timevarying Transition Probabilities". Business Cycles: Durations, Dynamics, and Forecasting, 144-165.

Gai, P., Haldane, A., \& Kapadia, S. (2011). "Complexity, Concentration and Contagion". Journal of Monetary Economics, 58(5), 453-470.

Garas, A., Argyrakis, P., Rozenblat, C., Tomassini, M., \& Havlin, S. (2010). Worldwide spreading of economic crisis. New Journal of Physics, 12(11), 113043.

Glasserman, P., \& Young, H. P. (2015). "How Likely is Contagion in Financial Networks?". Journal of Banking EF Finance, 50, 383-399.

Guidolin, M., \& Timmermann, A. (2007). "Asset Allocation under Multivariate Regime Switching". Journal of Economic Dynamics and Control, 31 (11), 35033544.

Haldane, A. G., \& May, R. M. (2011). "Systemic Risk in Banking Ecosystems". Nature, 469(7330), 351-355.

Hamilton, J. D. (1989). "A New Approach to the Economic Analysis of Nonstationary Time Series and the Business Cycle". Econometrica, 57(2), 357-384.

Hamilton, J. D. (2003). "Comment on "A Comparison of Two Business Cycle Dating Methods"". Journal of Economic Dynamics and Control, 27(9), 1691-1693.

Harding, D., \& Pagan, A. (2003a). "A Comparison of Two Business Cycle Dating Methods". Journal of Economic Dynamics and Control, 27(9), 1681-1690. 
Harding, D., \& Pagan, A. (2003b). "Rejoinder to James Hamilton". Journal of Economic Dynamics and Control, 27(9), 1695-1698.

Hooker, L. (2014). "The Seven Best Metaphors for the Economy". BBC News. Available at http://www/.bbc.com/news/business-30208476, accessed on August $15,2016$.

Kermack, W. O., \& McKendrick, A. G. (1927). "A Contribution to the Mathematical Theory of Epidemics". Proceedings of the Royal Society of London A: Mathematical, Physical and Engineering Sciences, 115(772), 700-721.

Lajmanovich, A., \& Yorke, J. A. (1976). "A Deterministic Model for Gonorrhea in a Nonhomogeneous Population". Mathematical Biosciences, 28(3), 221-236.

Moolgavkar, S. H., Day, N. E., \& Stevens, R. G. (1980). "Two-stage Model for Carcinogenesis: Epidemiology of Breast Cancer in Females". Journal of the National Cancer Institute, 65 (3), 559-569.

Peckham, R. (2013). "Economies of Contagion: Financial Crisis and Pandemic". Economy and Society, 42(2), 226-248.

Peng, R. D. (2008). "A Method for Visualizing Multivariate Time Series Data". Journal of Statistical Software, 25 (c01).

Shiller, R. J. (2008, August). "Infectious Exuberance". Available at www . theatlantic .com/magazine/archive/2008/07/infectious-exuberance/306839, accessed on October 28, 2016.

Shiller, R. J. (2014). "Speculative Asset Prices". The American Economic Review, $104(6), 1486-1517$.

Toivanen, M. (2013). "Contagion in the Interbank Network: An Epidemiological Approach". Bank of Finland Research Discussion Papers No. 19. Available at https://helda.helsinki.fi/bof/bitstream/handle/ 123456789/7981/172550. pdf? sequence=1, accessed on June 6, 2016. 


\section{Figures in text}

Figure 1: Visualization of dichotomous model, S=susceptible, I=infectious

(3)

(0)

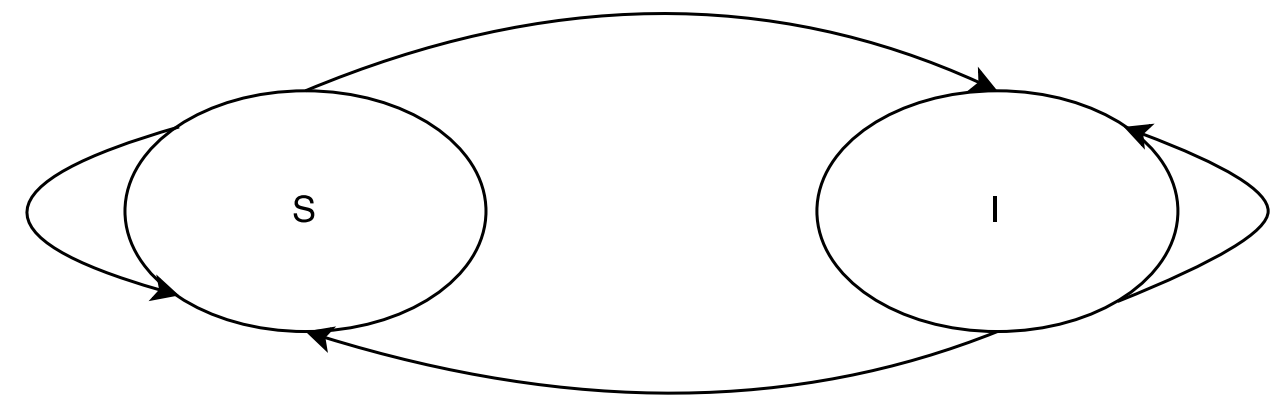

(2)

(1)

Figure 2: Visualization of two-level model with immediate recovery

(5)

(0)

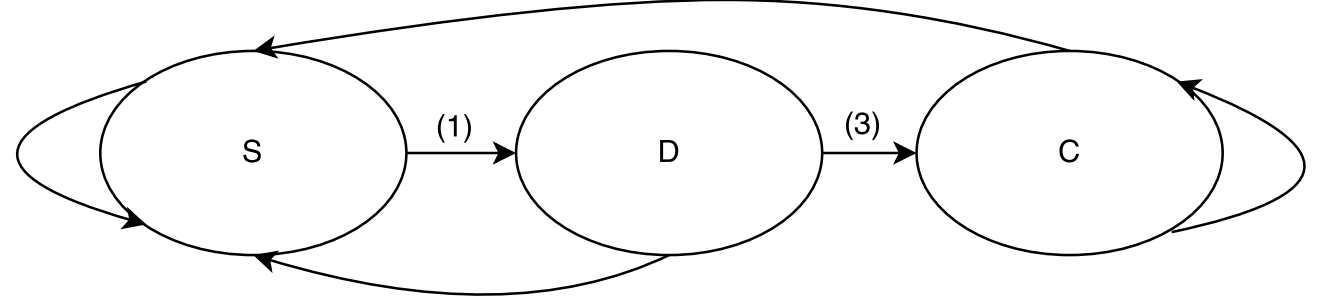

(2)

Figure 3: Visualization of two-level model with staged recovery

(5)

(0)

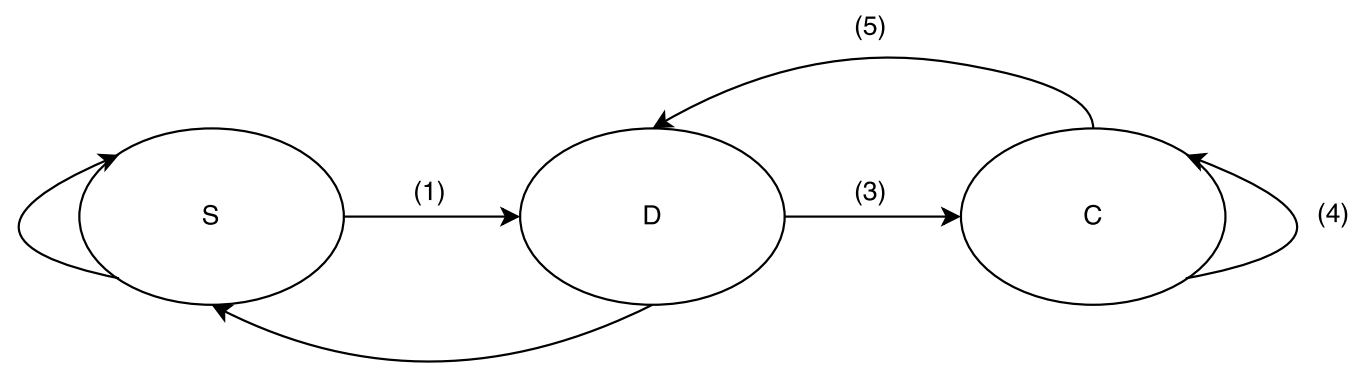

(2) 


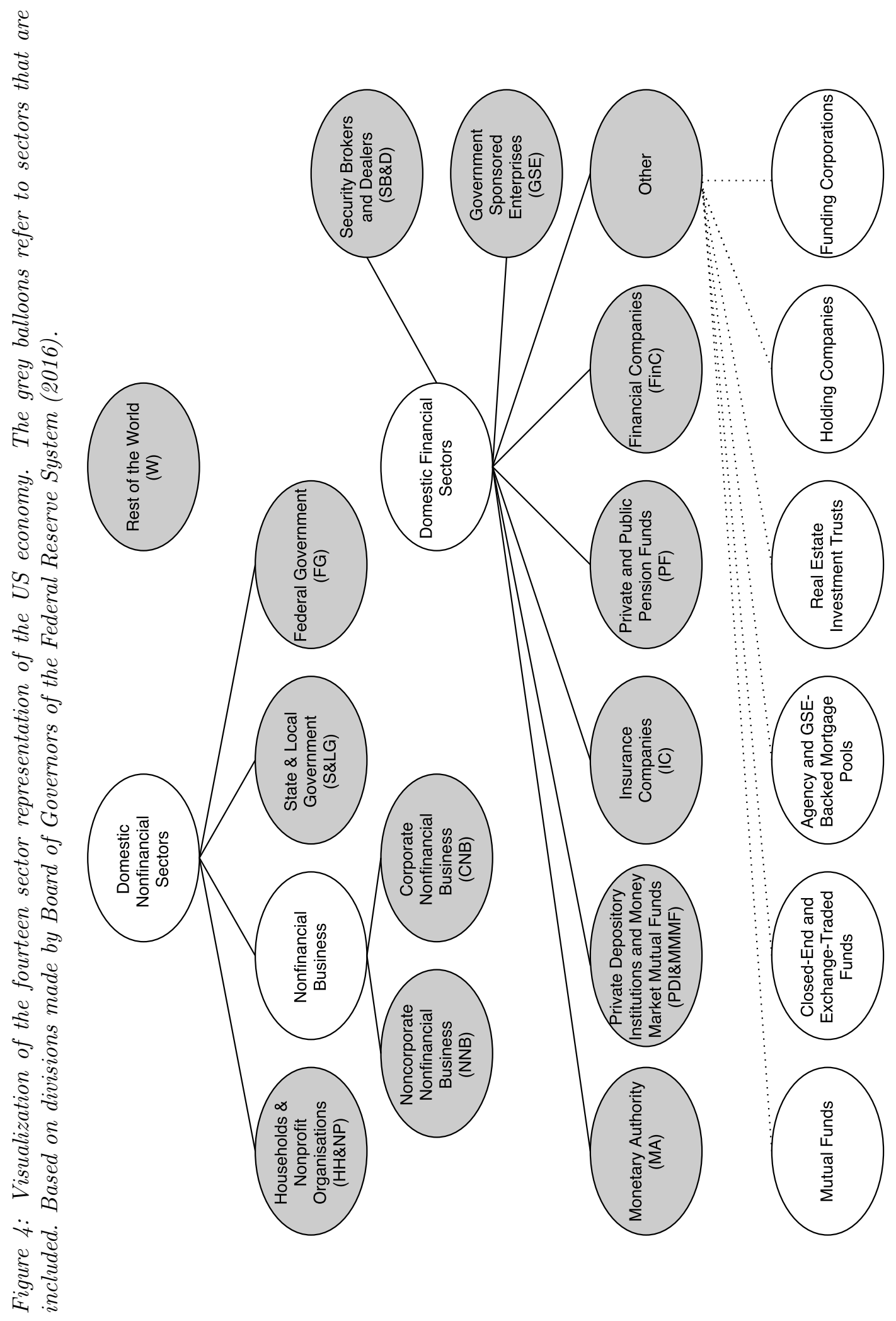



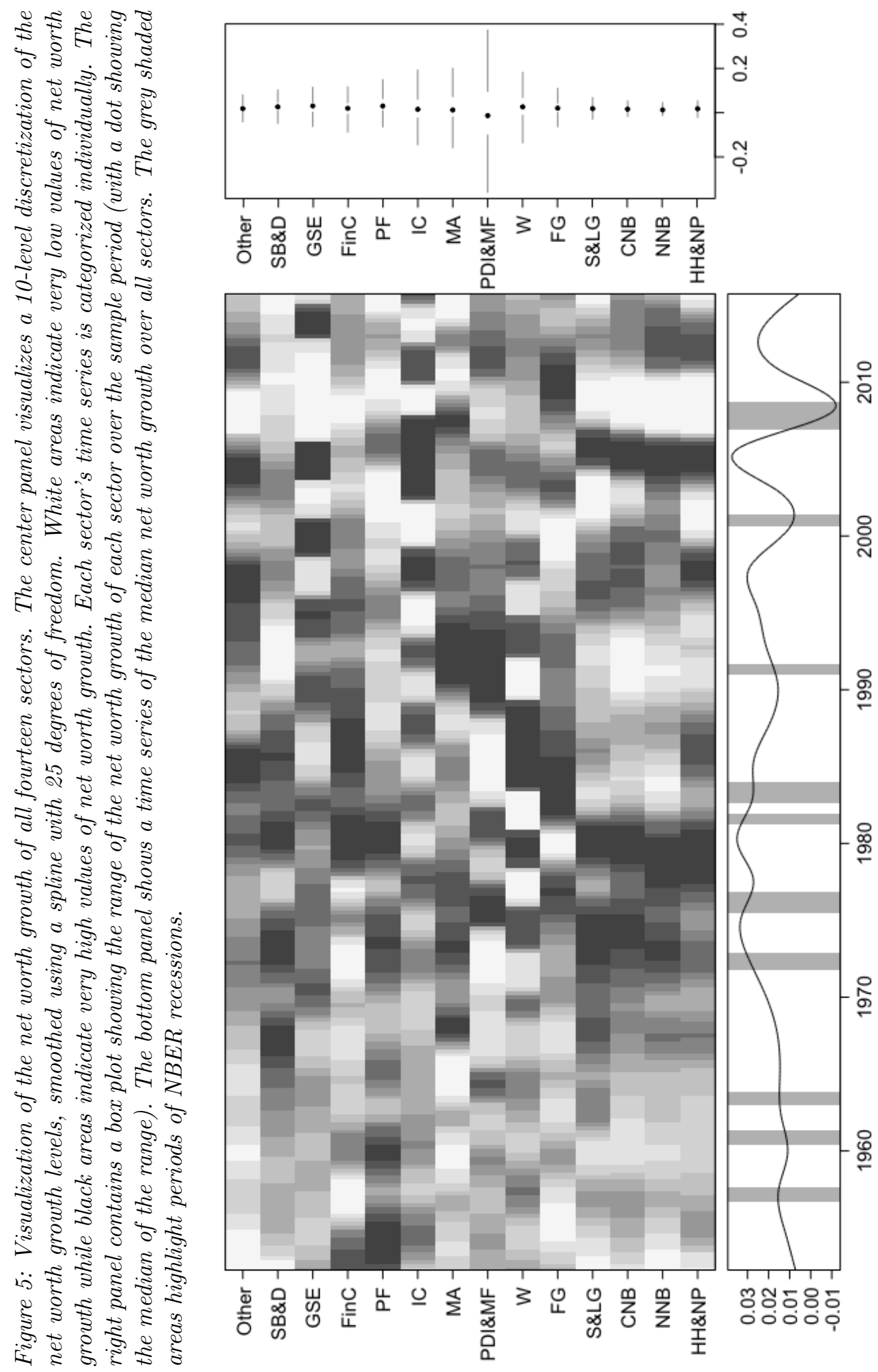
Figure 6: Epidemic curve, displaying the number of sectors (out of a total of 14) in downturn/crisis at each moment in time. Shaded grey areas represent crisis periods according to NBER recession dates.

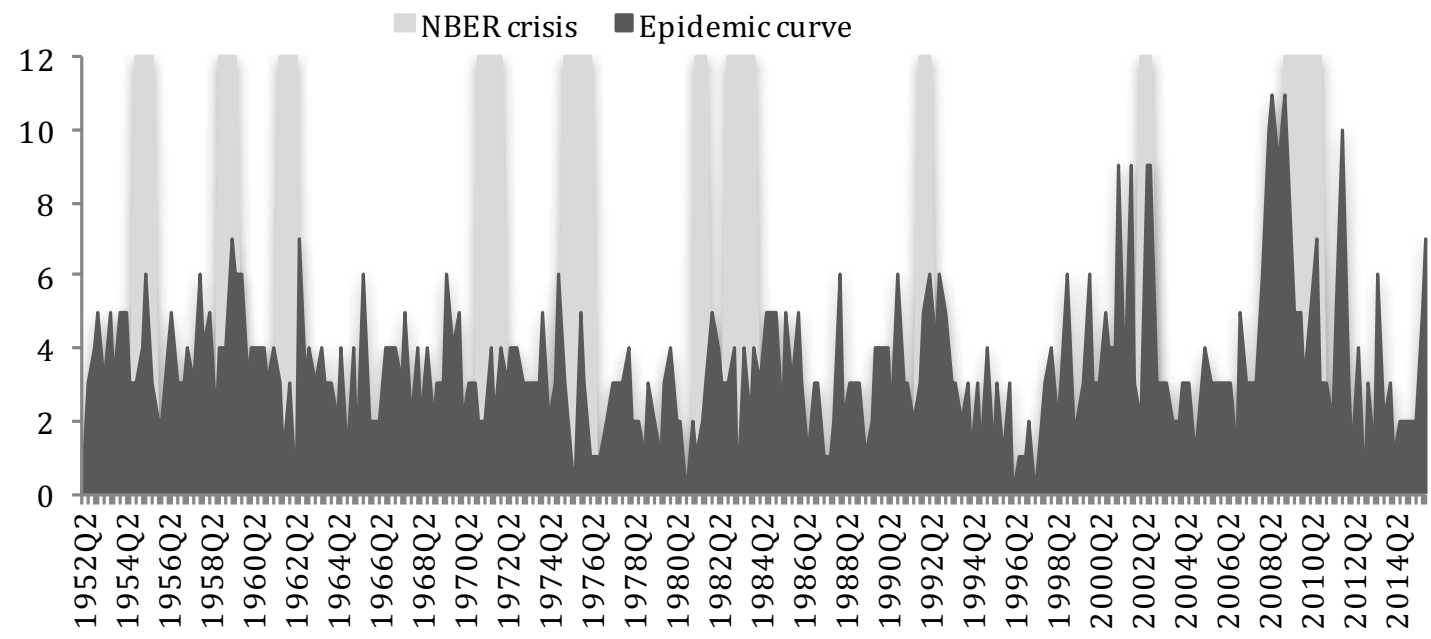

Figure 7: Different states of the fourteen sectors over time, according to the definition of the immediate recovery models. White areas indicate the respective sector is in a susceptible state, grey areas refer to downturns and black areas to crises.

Development of crises over time

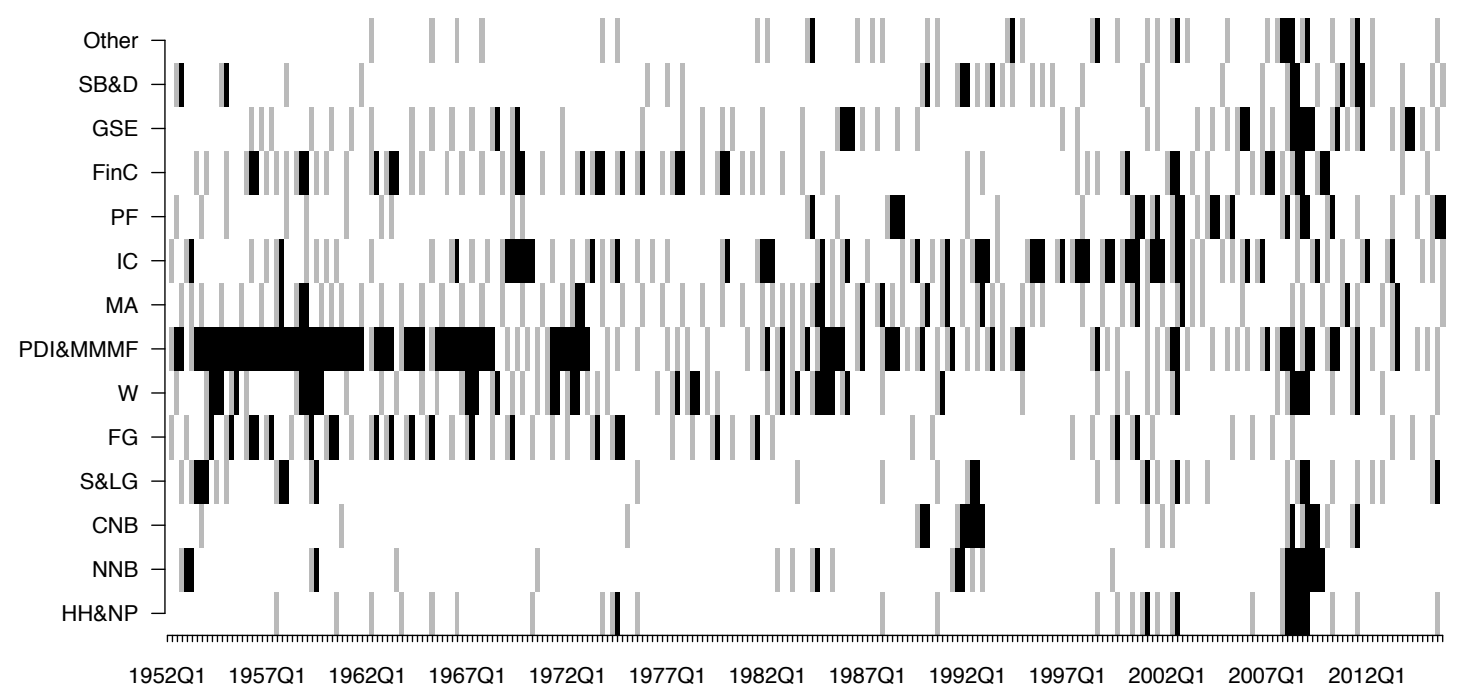




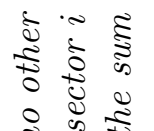

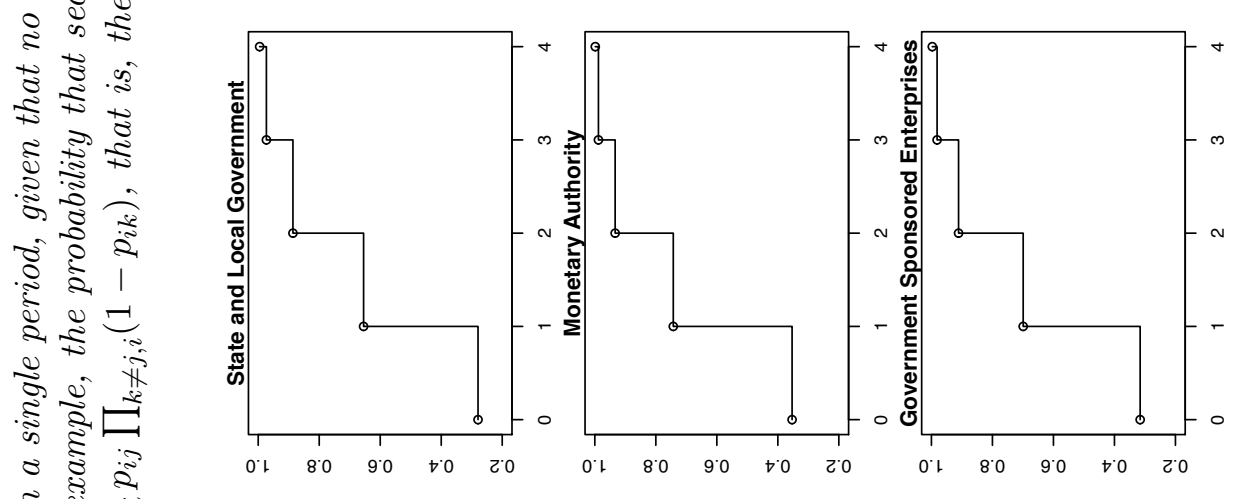

हల

के के

क्ष

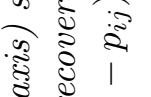

के है च

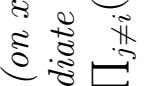

$\Rightarrow$ है

हี है
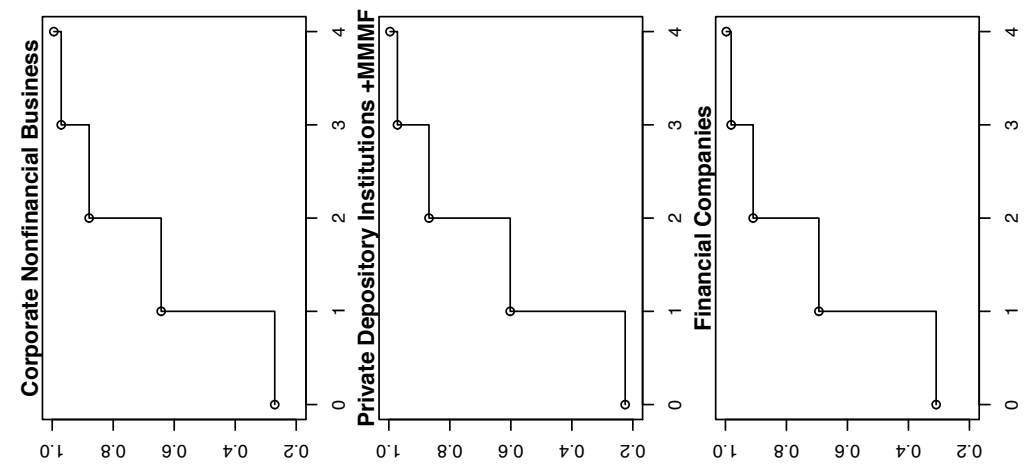

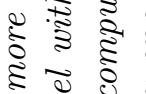

ฐ 8

अ है है

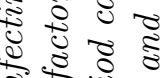

ปัँ

눤

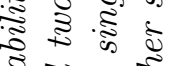

8 : 80
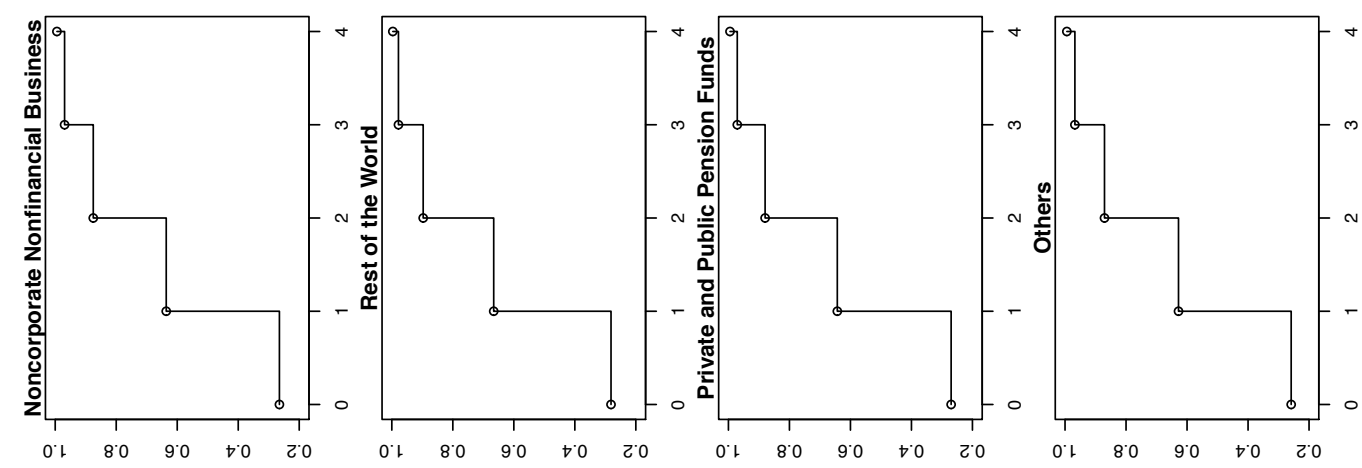

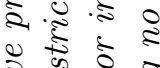

胥

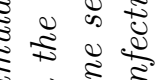

ปี. हิ हี

व क है

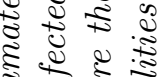

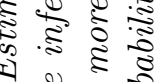

$\ddot{\infty}$ है ह है

हैं :
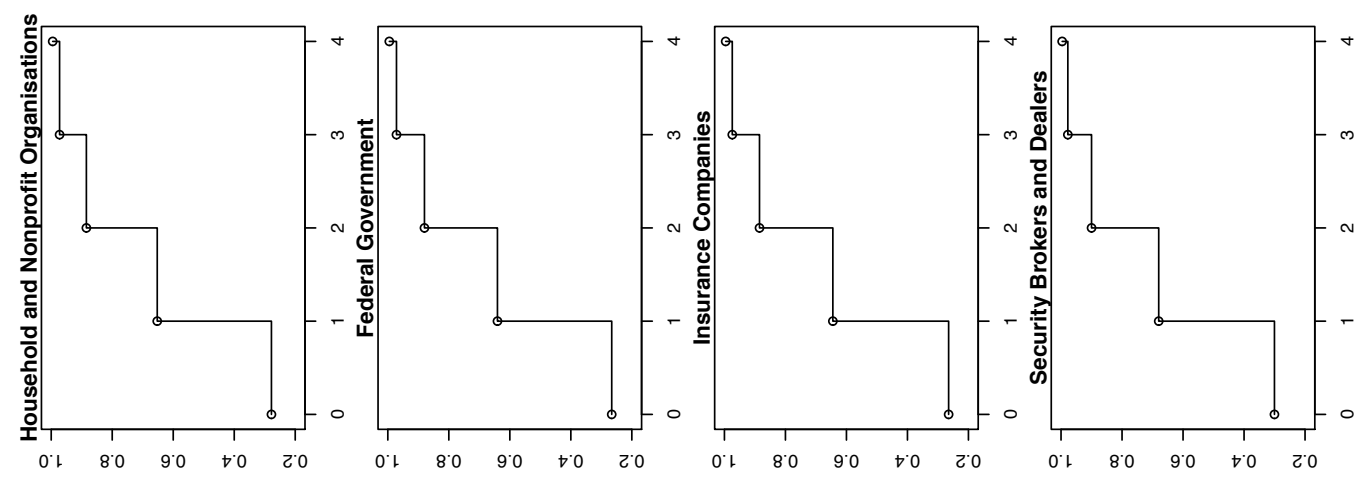


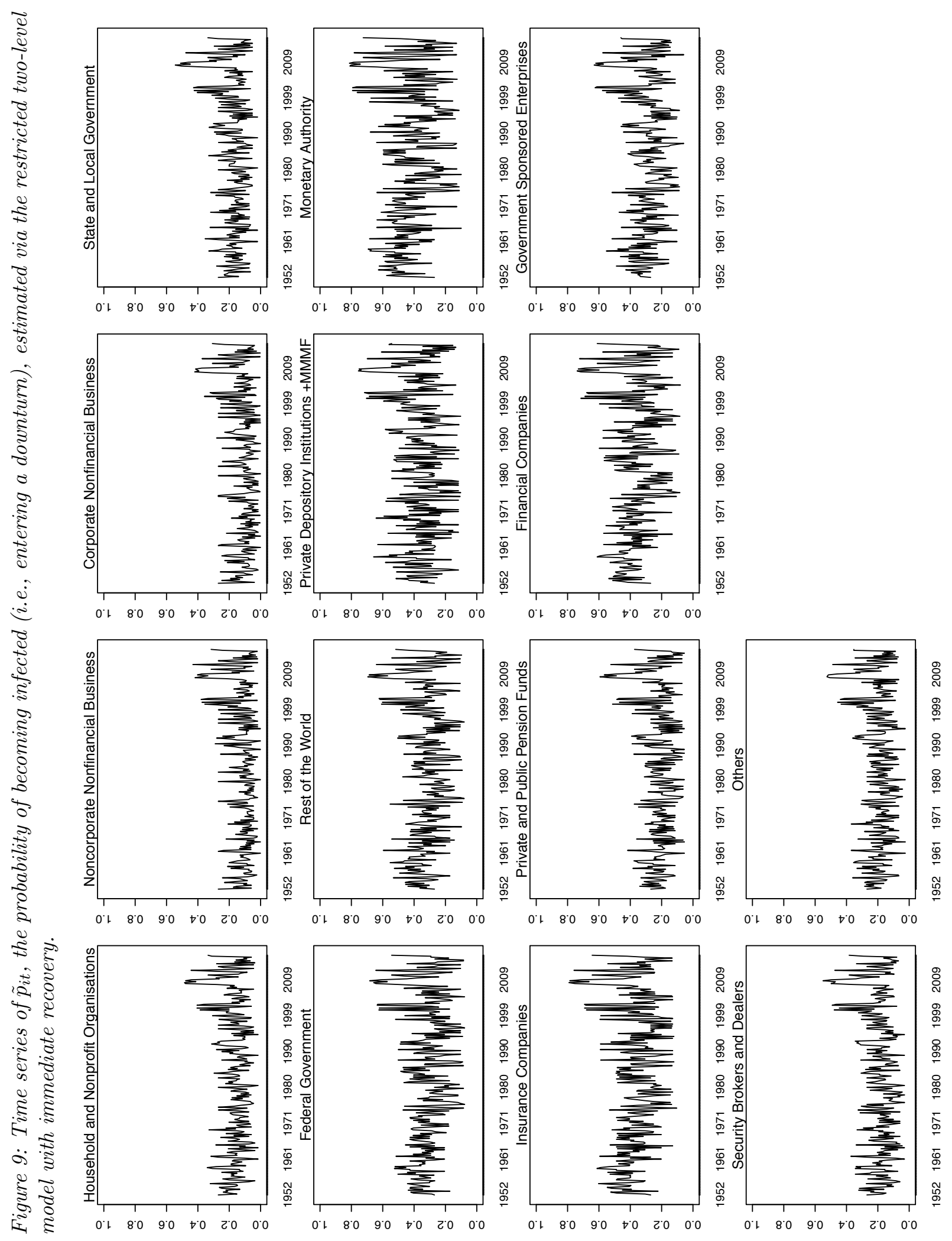




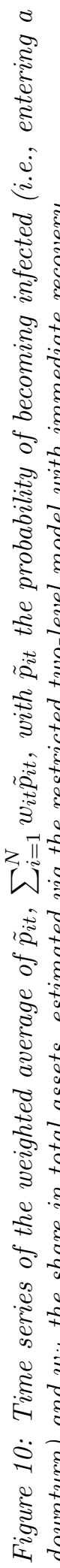

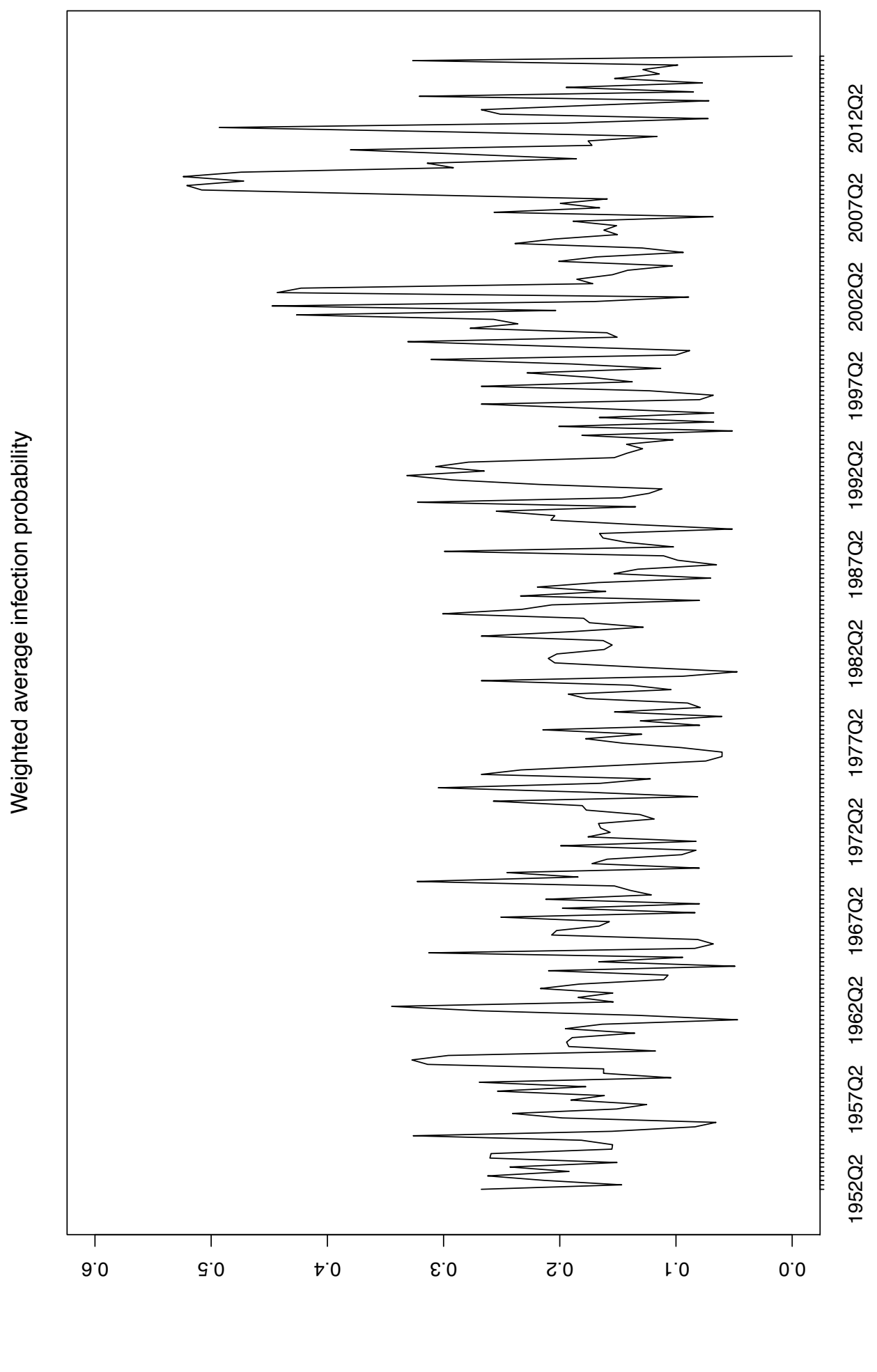




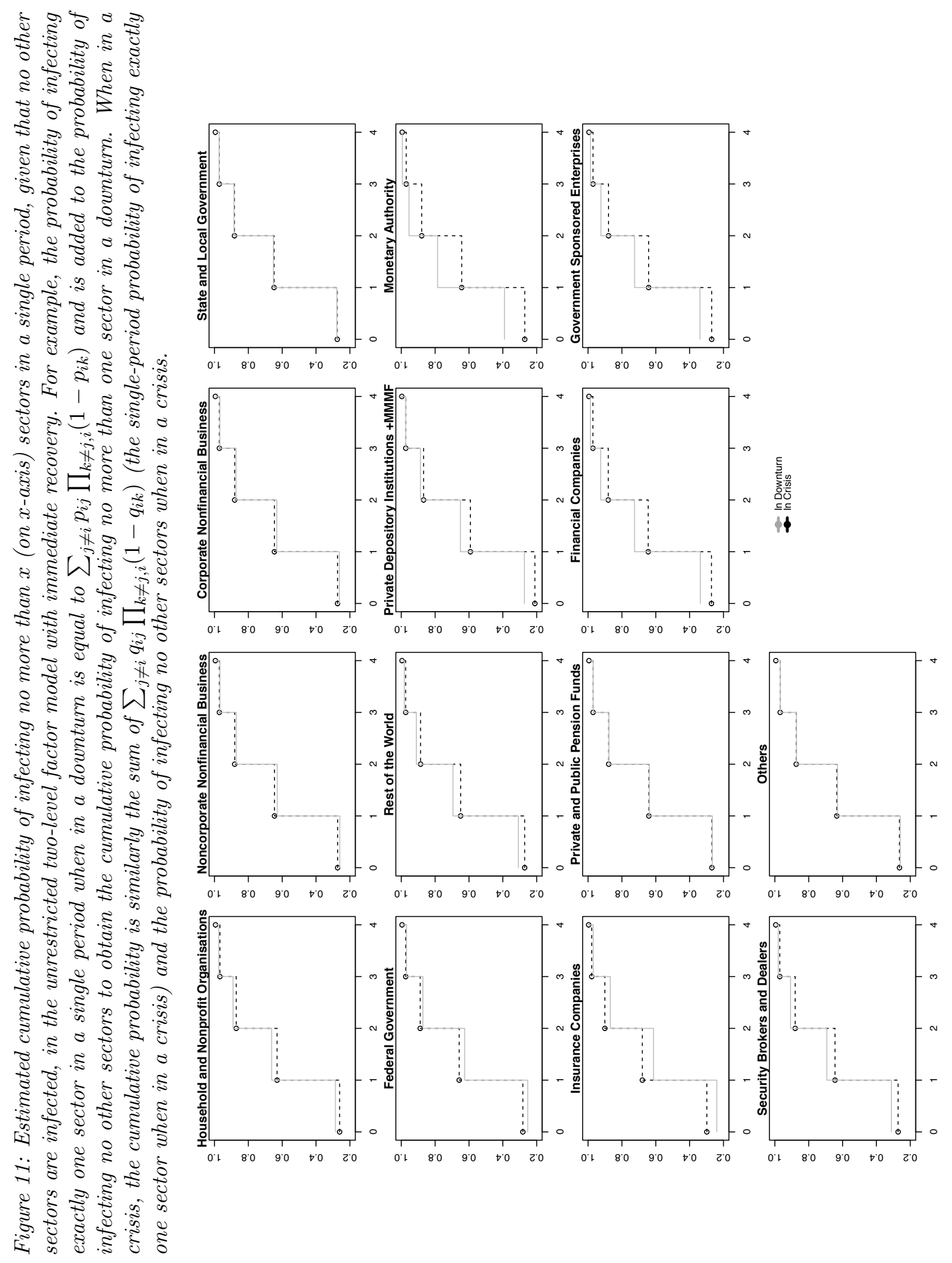




\section{Tables in text}

Table 1: Example demonstrating how the data would be labeled in the different model specifications described in section II: An epidemiological model of the US Economy. Net worth growth is defined as the growth in total assets - total liabilities of a single sector.

\begin{tabular}{|r|r|ccc|}
\hline Time period & $\Delta \%$ net worth & Dichotomous & Immediate recovery & Staged recovery \\
\hline 1. & 0.0130 & $\mathrm{~S}$ & $\mathrm{~S}$ & $\mathrm{~S}$ \\
2. & 0.0348 & $\mathrm{~S}$ & $\mathrm{~S}$ & $\mathrm{~S}$ \\
3. & -0.0214 & $\mathrm{I}$ & $\mathrm{D}$ & $\mathrm{D}$ \\
4. & 0.0038 & $\mathrm{~S}$ & $\mathrm{~S}$ & $\mathrm{~S}$ \\
5. & -0.0237 & $\mathrm{I}$ & $\mathrm{D}$ & $\mathrm{D}$ \\
6. & -0.0040 & $\mathrm{I}$ & $\mathrm{C}$ & $\mathrm{C}$ \\
7. & -0.0370 & $\mathrm{I}$ & $\mathrm{C}$ & $\mathrm{C}$ \\
8. & -0.0340 & $\mathrm{I}$ & $\mathrm{C}$ & $\mathrm{C}$ \\
9. & 0.0420 & $\mathrm{~S}$ & $\mathrm{~S}$ & $\mathrm{D}$ \\
10. & -0.0159 & $\mathrm{I}$ & $\mathrm{D}$ & $\mathrm{C}$ \\
11. & 0.0115 & $\mathrm{~S}$ & $\mathrm{~S}$ & $\mathrm{D}$ \\
12. & -0.0315 & $\mathrm{I}$ & $\mathrm{D}$ & $\mathrm{C}$ \\
\hline
\end{tabular}

Table 2: Summary statistics for the fourteen sectors for two-level epidemiological model with immediate recovery. $p_{i}$ is the recovery probability from a downturn $D, q_{i}$ the recovery probability from a crisis $C$. For a more detailed description of $p_{i}$ and $q_{i}$, consider probabilities (0)-(5) accompanying Figure 2. D refers to the downturn state, $C$ to a crisis. Total number of periods is 255. Note that the mean length of time in a crisis is equal to $1 / q_{i}$.

\begin{tabular}{|r|ccccc|}
\hline Column number & 1 & 2 & 3 & 4 & 5 \\
\hline & $\begin{array}{c}\text { number of } \\
\text { distinct } \\
\text { D episodes }\end{array}$ & $\begin{array}{c}\text { number of } \\
\text { periods } \\
\text { in C }\end{array}$ & $\begin{array}{c}\text { number of } \\
\text { distinct } \\
\text { C episodes }\end{array}$ & $\begin{array}{c}\text { Empirical } \\
p_{i}:\end{array}$ & $\begin{array}{c}\text { Empirical } \\
q_{i}:\end{array}$ \\
P(recover from D) & P(recover from C) \\
\hline HH\&NP & 23 & 8 & 4 & 0.826 & 0.500 \\
NNB & 13 & 14 & 5 & 0.615 & 0.357 \\
CNB & 12 & 12 & 5 & 0.583 & 0.417 \\
S\&LG & 25 & 13 & 8 & 0.680 & 0.615 \\
FG & 43 & 21 & 18 & 0.581 & 0.857 \\
W & 45 & 34 & 17 & 0.622 & 0.500 \\
PDI \& MMMF & 55 & 94 & 24 & 0.564 & 0.255 \\
MA & 70 & 16 & 13 & 0.814 & 0.813 \\
IC & 58 & 44 & 25 & 0.569 & 0.568 \\
PF & 29 & 18 & 11 & 0.621 & 0.611 \\
FinC & 53 & 27 & 16 & 0.698 & 0.593 \\
GSE & 44 & 16 & 8 & 0.818 & 0.500 \\
SB\&D & 30 & 11 & 8 & 0.733 & 0.727 \\
Other & 30 & 9 & 7 & 0.767 & 0.778 \\
\hline
\end{tabular}


Table 3: Number of times a sector immediately enters a downturn (D) after having recovered from a crisis $(C)$ ( $C$ followed by $S$ (susceptible) followed by $D$ ) compared to the total number of times a sector recovers from a crisis ( $C$ followed by $S$ ) according to the definitions of the two-level model with immediate recovery.

\begin{tabular}{|r|r|}
\hline Sector & Relapse probability \\
\hline Household and Nonprofit Organisations (HH\&NP) & 0.250 \\
Noncorporate Nonfinancial Business (NNB) & 0.200 \\
Corporate Nonfinancial Business (CNB) & 0.400 \\
State and Local Government (S\&LG) & 0.429 \\
Federal Government (FG) & 0.111 \\
Rest of the World (W) & 0.471 \\
Private Depository Institutions \& MMMF (PDI\&MMMF) & 0.542 \\
Monetary Authority (MA) & 0.462 \\
Insurance Companies (IC) & 0.400 \\
Private and Public Pension Funds (PF) & 0.300 \\
Financial Companies (FinC) & 0.313 \\
Government Sponsored Enterprises (GSE) & 0.375 \\
Security Brokers and Dealers (SB\&D) & 0.625 \\
Other & 0.429 \\
\hline
\end{tabular}

Table 4: Expected number of contaminations originating from the specific sector for the dichotomous model $\left(R_{0}=\frac{1}{p_{i}} \sum_{j \neq i} p_{i j}\right)$. Standard errors (in parentheses) computed via the delta method.

\begin{tabular}{|r|c|}
\hline Sector & $R_{0}$ \\
\hline Household and Nonprofit Organisations & $1.364(0.757)$ \\
Noncorporate Nonfinancial Business & $1.895(0.821)$ \\
Corporate Nonfinancial Business & $1.971(1.091)$ \\
State and Local Government & $1.628(0.770)$ \\
Federal Government & $1.919(0.460)$ \\
Rest of the World & $1.695(0.462)$ \\
Private Depository Institutions + MMMF & $2.418(0.454)$ \\
Monetary Authority & $1.092(0.307)$ \\
Insurance Companies & $2.063(0.390)$ \\
Private and Public Pension Funds & $1.842(0.617)$ \\
Financial Companies & $1.473(0.364)$ \\
Government Sponsored Enterprises & $1.282(0.386)$ \\
Security Brokers and Dealers & $1.452(0.616)$ \\
Other & $1.506(0.500)$ \\
\hline
\end{tabular}


整高

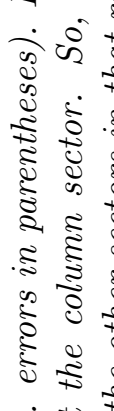

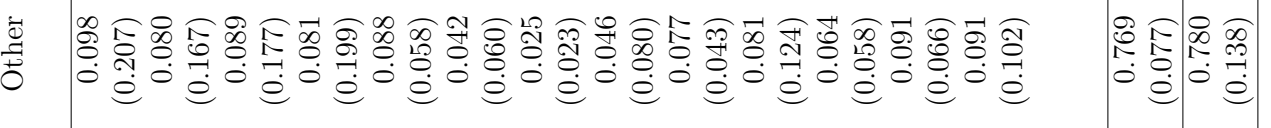

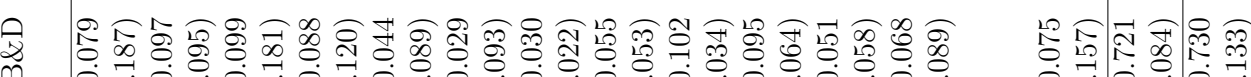

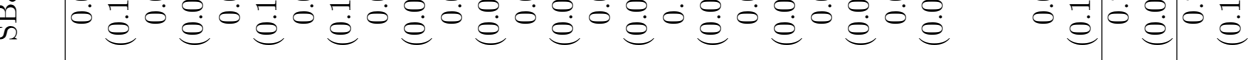

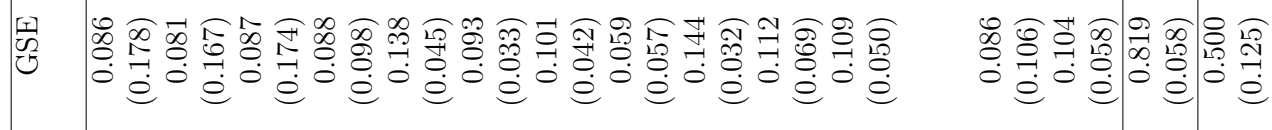

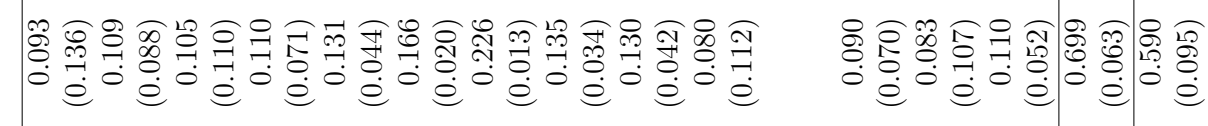

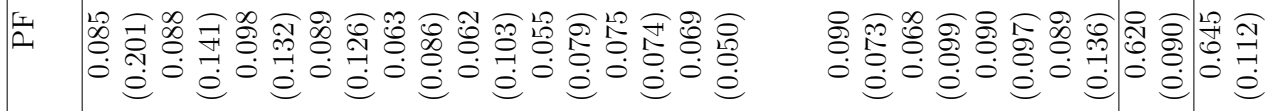

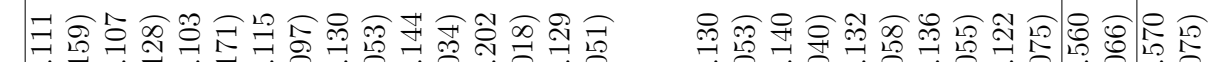

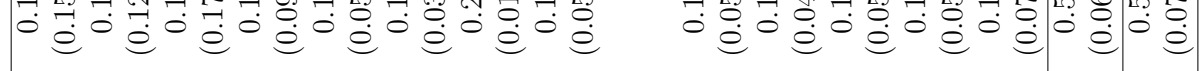

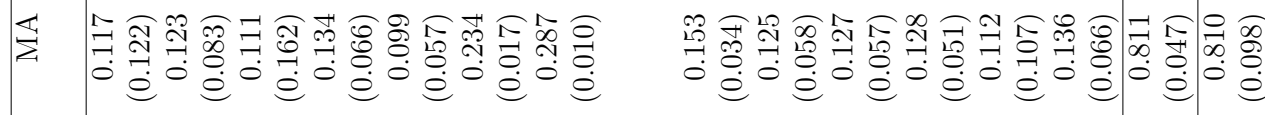

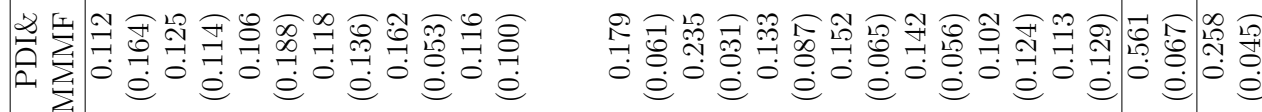

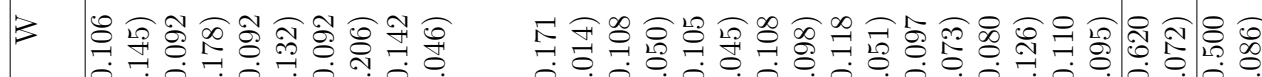

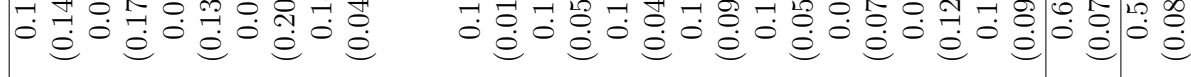

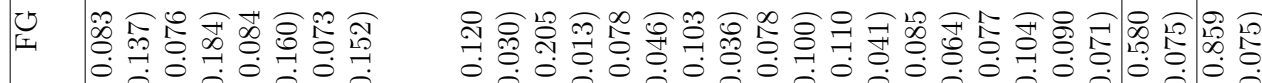

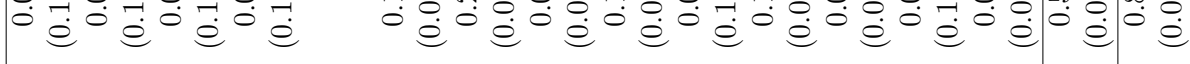

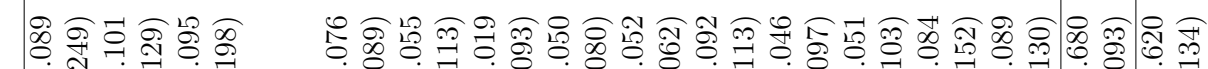

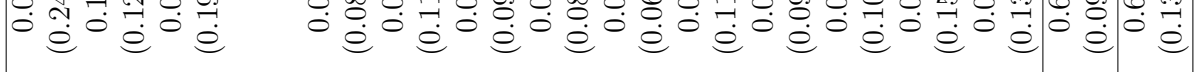

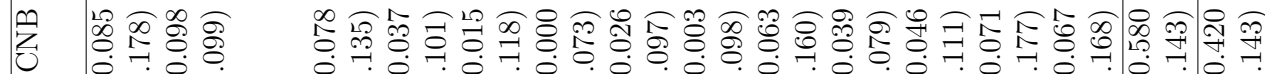
$\pi+$

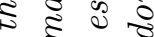

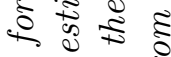

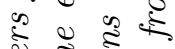

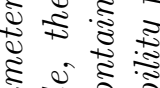

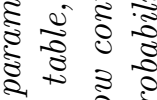

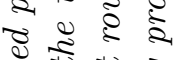

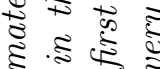

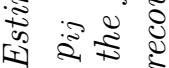

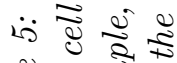

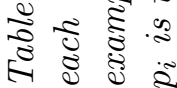

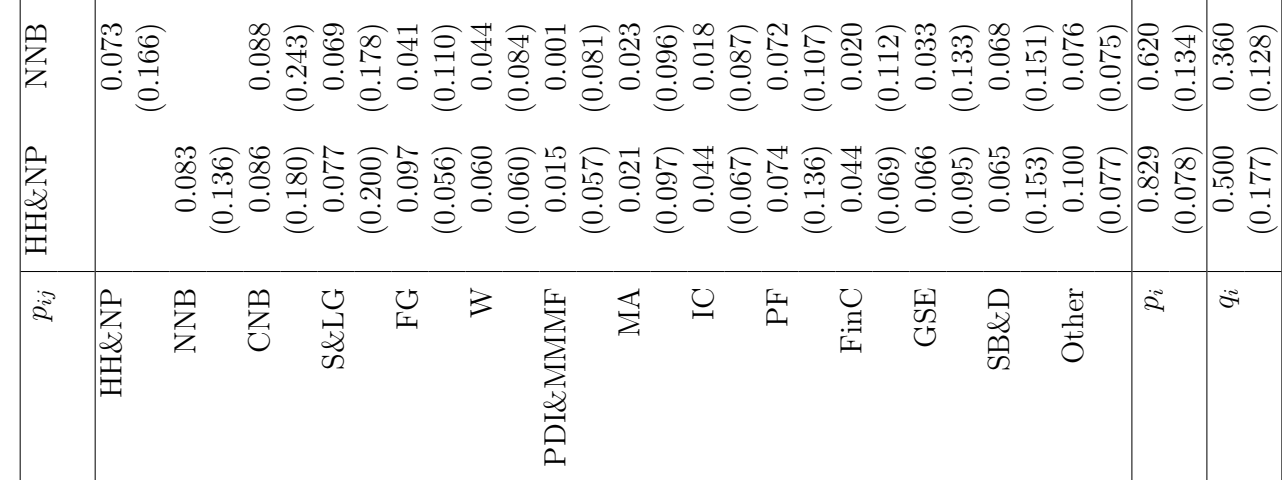


Table 6: Expected number of contaminations originating from the specific sector when in downturn $\left(R_{0}^{D}\right)$ and when in crisis $\left(R_{0}^{C}\right)$ for the restricted two-level model with immediate recovery, calculated using Equations 2 and 1, respectively. Standard errors (in parentheses) computed via the delta method.

\begin{tabular}{|r|ccc|}
\hline Sector & $R_{0}^{D}($ s.e. $)$ & $R_{0}^{C}($ s.e. $)$ \\
\hline Household and Nonprofit Organisations & $1.634(0.878)$ & $2.436(1.526)$ \\
Noncorporate Nonfinancial Business & $2.594(1.208)$ & $3.504(1.847)$ \\
Corporate Nonfinancial Business & $2.487(1.382)$ & $2.962(1.793)$ \\
State and Local Government & $1.838(0.823)$ & $1.956(0.943)$ \\
Federal Government & $1.857(0.404)$ & $1.451(0.327)$ \\
Rest of the World & $2.077(0.531)$ & $2.362(0.678)$ \\
Private Depository Institutions + MMMF & $3.614(0.726)$ & $5.186(1.157)$ \\
Monetary Authority & $1.214(0.320)$ & $1.214(0.346)$ \\
Insurance Companies & $2.189(0.399)$ & $2.169(0.449)$ \\
Private and Public Pension Funds & $1.974(0.629)$ & $1.927(0.667)$ \\
Financial Companies & $1.675(0.401)$ & $1.880(0.516)$ \\
Government Sponsored Enterprises & $1.491(0.449)$ & $2.192(0.824)$ \\
Security Brokers and Dealers & $1.585(0.637)$ & $1.571(0.676)$ \\
Other & $1.661(0.516)$ & $1.644(0.570)$ \\
\hline & & \\
\hline
\end{tabular}

Table 7: Expected number of contaminations originating from the specific sector when in downturn $\left(R_{0}^{D}\right)$ and when in crisis $\left(R_{0}^{C}\right)$ for the unrestricted two-level model with immediate recovery, calculated using Equations 4 and 3, respectively. No standard errors available, because of lack of data on some interactions between sectors.

\begin{tabular}{r|cc|}
\hline Sector & $R_{0}^{D}$ & $R_{0}^{C}$ \\
\hline Household and Nonprofit Organisations & 1.626 & 2.384 \\
Noncorporate Nonfinancial Business & 2.583 & 3.552 \\
Corporate Nonfinancial Business & 2.507 & 3.028 \\
State and Local Government & 1.854 & 1.966 \\
Federal Government & 1.873 & 1.495 \\
Rest of the World & 2.038 & 2.216 \\
Private Depository Institutions + MMMF & 3.522 & 4.697 \\
Monetary Authority & 1.169 & 1.083 \\
Insurance Companies & 2.200 & 2.307 \\
Private and Public Pension Funds & 1.985 & 1.932 \\
Financial Companies & 1.658 & 1.743 \\
Government Sponsored Enterprises & 1.476 & 2.057 \\
Security Brokers and Dealers & 1.587 & 1.523 \\
Other & 1.650 & 1.637 \\
\hline
\end{tabular}


Table 8: Expected number of contaminations originating from the specific sector when in downturn $\left(R_{0}^{D}\right)$ and when in crisis $\left(R_{0}^{C}\right)$ for the restricted two-level model with staged recovery, calculated using Equations 3 and 5, respectively. Standard errors (in parentheses) computed via the delta method.

\begin{tabular}{|c|c|c|}
\hline Sector & (s.e.) & (s.e.) \\
\hline Household and Nonprofit Organisations & $1.951(0.804)$ & $2.390(1.211)$ \\
\hline Noncorporate Nonfinancial Business & $3.945(1.335)$ & $3.273(1.170)$ \\
\hline Corporate Nonfinancial Business & $4.152(2.370)$ & $2.891(1.671)$ \\
\hline State and Local Government & $2.547(1.153)$ & $1.792(0.846)$ \\
\hline Federal Government & $2.555(0.383)$ & $1.254(0.178)$ \\
\hline Rest of the World & $2.640(0.700)$ & $1.827(0.499)$ \\
\hline vate Depository Institutions + MMMF & $4.459(1.201)$ & $3.539(0.898)$ \\
\hline y Authority & $1.235(0.023)$ & $0.904(0.083)$ \\
\hline Companies & $3.289(0.681)$ & $1.874(0.390)$ \\
\hline Private and Public Pension Funds & $2.803(0.931)$ & $1.763(0.596)$ \\
\hline Financial Companies & $2.144(0.156)$ & $1.614(0.190)$ \\
\hline Government Spo & $1.696(0.450)$ & $1.972(0.645)$ \\
\hline Security Brokers and Dealers & $2.187(0.766)$ & $1.502(0.544)$ \\
\hline Other & $2.024(0.449)$ & $1.532(0.381)$ \\
\hline
\end{tabular}





\section{An Epidemiological Model of Crisis Spread Across Sectors in the United States}

SUPPLEMENTAL APPENDICES (FOR ONLINE PUBLICATION ONLY)

Eva F. Janssens, Robin L. Lumsdaine and Sebastiaan H.L.C.G. Vermeulen 


\section{Table of Contents}

Appendix A: Modified model assumptions for the two-level model 2

Appendix B: Derivation of the likelihood function, gradient, and Hessian 4

Appendix C: Summary statistics of the data 8

Appendix D: Model parameter estimates 9

D1: Dichotomous model 9

D2: Unrestricted two-level model with immediate recovery $\quad 10$

D3: Two-level model with staged recovery 11

Appendix E: Robustness $\quad 12$

E1: Robustness over time 12

E2: Robustness to the crisis definition 16

E3: Robustness to seasonal variation 19 


\section{A Modified model assumptions for the two-level model}

In this section, the modeling assumptions of the two-level model are discussed, since they are slightly different from the dichotomous model. The base case that is discussed is for the two-level model with immediate recovery. Where the discussion would differ for the staged recovery model, this is mentioned in a footnote.

1. The world consists of a pre-specified constant number of sectors, $N$. Each sector has its own recovery rate and rate of interaction with other sectors. It is possible to aggregate sectors together if there are reasons to believe they are homogeneous (i.e. same transmission probabilities and recovery probabilities), and thus reduce the model dimension.

2. A sector can be in one of three states: (1) susceptible (i.e., not infected), (2) downturn, or (3) crisis. Unlike in many epidemiological models of disease, in the economic context, a sector cannot become immune.

3. A sector is susceptible as long as the growth of its net worth is positive. A sector is in state $D$ (downturn) as soon as it has had one period of negative net worth growth, where net worth is defined as total assets - total liabilities for that sector. A sector is in state $C$ (crisis) after two consecutive periods of negative net worth growth. It is contagious both in the downturn and crisis states, until its net worth growth turns positive again, after which it enters the susceptible state again ${ }^{1}$. For a numerical example, consider the fourth (fifth for staged recovery) column of Table 1.

4. Downturns and crises are spread from sector-to-sector.

5. In every time period (which is set to the length of a quarter due to the frequency of the data) it is possible for a sector to change states once (e.g., become susceptible or enter a downturn or crisis). By construction, it is assumed that recovery cannot occur in the same period as infection; more specifically, the frequency of the data do not enable observation of infection/recovery episodes that occur within a single time period.

6. The probability that a susceptible sector $j$ gets contaminated by an infectious sector (a sector in state $D$ or $C$ ) $i$ is denoted by $p_{i j}$ and satisfies the Markov property, that is, it is independent of both the time since sector $j$ was last susceptible and the time since $i$ was last infectious. An implication of the Markov assumption is that the length until infection of sector $j$ by infected sector $i$ therefore follows a geometric distribution.

\footnotetext{
${ }^{1}$ In the staged-recovery model, once a sector enters a crisis, it does not leave it at once - it first enters the downturn state again. Only after it has experienced two consecutive periods of positive net worth growth does it enter the susceptible state again.
} 
7. The recovery probability is also assumed to be first-order Markov, that is, the probability that an infected sector recovers and thus changes states from $\mathrm{D}$ to $\mathrm{S}$ or from $\mathrm{C}$ to $\mathrm{D}$ in a period is independent of the number of periods it has already been infected. The (sector-specific) probability that sector $i$ will recover next period from a downturn (D) is denoted by $p_{i}$; therefore the probability that sector $i$ enters a crisis is $\left(1-p_{i}\right)$. The probability of recovering next period from a crisis is denoted by $q_{i}$. The mean time spent in the crisis period is $1 / q_{i}$. The probability that sector $i$ is still in a crisis after $T$ periods is therefore $\left(1-q_{i}\right)^{T}$, i.e., the probability is decreasing in $T$. Note that since we are using a discrete modeling framework, the Markov property implies that the length of the crisis period follows a geometric distribution, which is the discrete counterpart to the exponential distribution as used by Becker and Angulo (1981).

8. $R_{0}$ is the basic reproduction number, a commonly used statistic in epidemiology. It is equal to the expected number of infections that one infectious sector will cause in an otherwise purely susceptible system, that is, a system where no other sectors are infected (i.e., all other states are in state $\mathrm{S}$ ). Since a sector is infectious in both states $D$ and $C$, we can write down two different reproduction numbers. $R_{0}$ for sector $i$ is equal to

$$
\begin{aligned}
& R_{0}^{C}=\frac{1}{q_{i}} \sum_{j \neq i} p_{i j} \\
& R_{0}^{D}=\sum_{j \neq i} p_{i j}+\left(1-p_{i}\right) \frac{1}{q_{i}} \sum_{j \neq i} p_{i j}
\end{aligned}
$$

with $R_{0}^{D}$ the basic reproduction number once a sector has entered a downturn and $R_{0}^{C}$ the basic reproduction number once a sector has entered a crisis. ${ }^{2}$ Here it is assumed that contacts are independent, so that the probability of sector $i$ being contaminated by sector $j$ does not change if sector $i$ has also contaminated sector $k$ in that same period. This assumption implies the following: if sector $i$ already has contaminated sector $j$ in one period, the probability that sector $i$ will also contaminate sector $k$, denoted as $p_{i k}$, in that same period does not change.

\footnotetext{
${ }^{2}$ For the staged recovery model, $R_{0}^{D}$ changes. The expected number of times a sector will enter a crisis from the moment it enters a downturn is given by $\frac{1-p_{i}}{p_{i}}$ (the expected value of a geometric distribution starting at zero). Every period it is in a downturn, it is expected to infect $\sum_{j \neq i} p_{i j}$ other sectors (assuming no one is infected yet) and will on average be in a downturn for $\left(\frac{1}{p_{i}}\right)$ periods. Once it enters a crisis, it will on average infect $\frac{1}{q_{i}} \sum_{j \neq i} p_{i j}$ other sectors (assuming no sectors are infected yet). Thus, this gives us
}

$$
R_{0}^{D}=\frac{1-p_{i}}{p_{i}} \frac{1}{q_{i}} \sum_{j \neq i} p_{i j}+\frac{1}{p_{i}} \sum_{j \neq i} p_{i j}
$$




\section{B Derivation of the likelihood function, gradient, and Hessian}

In this section, the likelihood function of the two-level epidemiological model with immediate recovery, its gradient and Hessian are derived. In Equation 7, the likelihood for sector $i$ at time $t$ is given. In this equation, $\tilde{p}_{j t}=1-\prod_{j \in D_{t}, C_{t}}\left(1-p_{j i}\right)$. To obtain the full likelihood value for all sectors and all observations in time, multiply all $\mathscr{L}_{i t}$ for $t=1, \ldots, T$ and $i=1, \ldots, N$. The log-likelihood value for all sectors and observations is therefore the sum of the log of $\mathscr{L}_{i t}$ for $t=1, \ldots, T$ and $i=1, \ldots, N$. The natural logarithm of $\mathscr{L}_{i t}$ will from this point be denoted as $l_{i t}$.

$$
l_{i, t}= \begin{cases}\log \left(1-\tilde{p}_{i t}\right) & \text { if } X_{i t}=S \text { and } X_{i t-1}=S \\ \log \left(\tilde{p}_{i t}\right) & \text { if } X_{i t}=D \text { and } X_{i t-1}=S \\ \log \left(p_{i}\right) & \text { if } X_{i t}=S \text { and } X_{i t-1}=D \\ \log \left(1-p_{i}\right) & \text { if } X_{i t}=C \text { and } X_{i t-1}=D \\ \log \left(q_{i}\right) & \text { if } X_{i t}=S \text { and } X_{i t-1}=C \\ \log \left(1-q_{i}\right) & \text { if } X_{i t}=C \text { and } X_{i t-1}=C\end{cases}
$$

To obtain the gradient of the log-likelihood value, we must take the derivative of $l_{i t}$ for all parameters $\theta$, resulting in vector $d\left(l_{i t}\right) / d(\theta)$ and take the sum of $d\left(l_{i t}\right) / d(\theta)$ for all $t=1, \ldots, T$ and $i=1, \ldots, N$.

$$
\begin{aligned}
& \frac{\delta l_{i t}}{\delta p_{i}}= \begin{cases}\frac{1}{p_{i}} & \text { if } X_{i t}=S \text { and } X_{i t-1}=D \\
-\frac{1}{1-p_{i}} & \text { if } X_{i t}=C \text { and } X_{i t-1}=D \\
0 & \text { else }\end{cases} \\
& \frac{\delta l_{i t}}{\delta q_{i}}= \begin{cases}\frac{1}{q_{i}} & \text { if } X_{i t}=S \text { and } X_{i t-1}=C \\
-\frac{1}{1-q_{i}} & \text { if } X_{i t}=C \text { and } X_{i t-1}=C \\
0 & \text { else }\end{cases}
\end{aligned}
$$




$$
\begin{aligned}
& \frac{\delta l_{i t}}{\delta p_{j i}}= \begin{cases}\frac{\delta \log \tilde{p}_{i t}}{\delta p_{j i}} & \text { if } X_{i t}=D \text { and } X_{i t-1}=S \\
\frac{\delta \log \left(1-\tilde{p}_{i t}\right)}{\delta p_{j i}} & \text { if } X_{i t}=S \text { and } X_{i t-1}=S \\
0 & \text { else }\end{cases} \\
& \frac{\delta \log \tilde{p}_{i t}}{\delta p_{j i}}= \begin{cases}\frac{1-\tilde{p}_{i t}}{\tilde{p}_{i t}\left(1-p_{j i}\right)} & \text { if } X_{j t-1}=D \text { or } C \\
0 & \text { if } X_{j t-1}=S\end{cases} \\
& \frac{\delta \log \left(1-\tilde{p}_{i t}\right)}{\delta p_{j i}}= \begin{cases}-\frac{1}{1-p_{j i}} & \text { if } X_{j t-1}=D \text { or } C \\
0 & \text { if } X_{j t-1}=S\end{cases}
\end{aligned}
$$

For the Hessian matrix, we need the following building blocks, which follow directly from the gradient. Before writing out the more important formulas of the Hessian, we should already note that a lot of elements in the gradient are zero. In these cases, the second derivatives also will be zero; these elements are omitted from the formulas below. Similarly, when the parameter to which the partial derivative is taken does not appear in the gradient, the second derivative will also be zero, so these elements are also omitted. Again, to obtain the full Hessian matrix for all observations and sectors, one should take the sum over all $t=1, \ldots, T$ and $i=1, \ldots, N$.

$$
\begin{aligned}
& \frac{\delta^{2} l_{i t}}{\delta p_{i}^{2}}= \begin{cases}-\frac{1}{p_{i}^{2}} & \text { if } X_{i t}=S \text { and } X_{i t-1}=D \\
-\frac{1}{\left(1-p_{i}\right)^{2}} & \text { if } X_{i t}=C \text { and } X_{i t-1}=D \\
0 & \text { else }\end{cases} \\
& \frac{\delta^{2} l_{i t}}{\delta q_{i}^{2}}= \begin{cases}-\frac{1}{q_{i}^{2}} & \text { if } X_{i t}=S \text { and } X_{i t-1}=C \\
-\frac{1}{\left(1-q_{i}\right)^{2}} & \text { if } X_{i t}=C \text { and } X_{i t-1}=C \\
0 & \text { else }\end{cases} \\
& \frac{\delta^{2} l_{i t}}{\delta p_{j i}^{2}}= \begin{cases}\frac{\delta^{2} \log \tilde{p}_{i t}}{\delta p_{j i}^{2}} & \text { if } X_{i t}=D \text { and } X_{i t-1}=S \\
\frac{\delta^{2} \log \left(1-\tilde{p}_{i t}\right)}{\delta p_{j i}^{2}} & \text { if } X_{i t}=S \text { and } X_{i t-1}=S \\
0 & \text { else }\end{cases}
\end{aligned}
$$




$$
\begin{aligned}
& \frac{\delta^{2} \log \tilde{p}_{i t}}{\delta p_{j i}^{2}}= \begin{cases}\frac{\left(1-\tilde{p}_{i t}\right)^{2}}{\left(1-p_{j i}\right)^{2} \tilde{p}_{i t}^{2}} & \text { if } X_{j t-1}=D \text { or } C \\
0 & \text { else }\end{cases} \\
& \frac{\delta^{2} \log \left(1-\tilde{p}_{i t}\right)}{\delta p_{j i}^{2}}= \begin{cases}-\frac{1}{\left(1-p_{j i}\right)^{2}} & \text { if } X_{j t-1}=D \text { or } C \\
0 & \text { if } X_{j t-1}=S\end{cases} \\
& \frac{\delta^{2} l_{i t}}{\delta p_{j i} p_{k i}}= \begin{cases}\frac{\delta^{2} \log \tilde{p}_{i t}}{\delta p_{j i} p_{k i}} & \text { if } X_{i t}=D \text { and } X_{i t-1}=S \\
\frac{\delta^{2} \log \left(1-\tilde{p}_{i t}\right)}{\delta p_{j i} p_{k i}} & \text { if } X_{i t}=S \text { and } X_{i t-1}=S \\
0 & \text { else }\end{cases} \\
& \frac{\delta^{2} \log \tilde{p}_{i t}}{\delta p_{j i} p_{k i}}= \begin{cases}-\frac{\left(1-\tilde{p}_{i t}\right)}{\tilde{p}_{i t}^{2}\left(1-p_{k i}\right)\left(1-p_{j i}\right)} & \text { if } X_{j t-1}=D \text { or } C \\
0 & \text { else }\end{cases} \\
& \frac{\delta^{2} \log 1-\tilde{p}_{i t}}{\delta p_{j i} p_{k i}}=0
\end{aligned}
$$

In the code which is provided via an online appendix, one can see that an additional parameter has been introduced, $n$ (stands for 'nature'), which is introduced to tackle the issue that at some periods, no sector might be in a crisis, so the probability of entering a crisis endogenously would be zero. In that case, a sector can still enter a crisis, not because of contamination via the other sectors, but due to a sudden act of nature (i.e., exogenously). So in case no sector is in a crisis, $\tilde{p}_{i t}$ is replaced by $n$. In other words, a sector can become infected either from another sector or exogenously ('nature'). This perturbation is necessary for the model to avoid reaching a steady (non-infected) state. The first derivative of $\log \tilde{p}_{i t}$ to $n$ is given by:

$$
\frac{\partial \log \tilde{p}_{i t}}{\partial n}=\left\{\begin{array}{ll}
1 / n & \text { if no sectors contaminated } \\
0 & \text { else }
\end{array} .\right.
$$

The second derivative of $\log \tilde{p}_{i t}$ with respect to $n$ is given by:

$$
\frac{\partial^{2} \log \tilde{p}_{i t}}{\partial n^{2}}= \begin{cases}-1 / n^{2} & \text { if no sectors contaminated } \\ 0 & \text { else. }\end{cases}
$$


The first derivative of $\log \left(1-\tilde{p}_{i t}\right)$ to $n$ is given by

$$
\frac{\partial \log \left(1-\tilde{p}_{i t}\right)}{\partial n}= \begin{cases}-1 /(1-n) & \text { if no sectors contaminated } \\ 0 & \text { else. }\end{cases}
$$

The second derivative of $\log \left(1-\tilde{p}_{i t}\right)$ to $n$

$$
\frac{\partial^{2} \log \left(1-\tilde{p}_{i t}\right)}{\partial n^{2}}= \begin{cases}-1 /(1-n)^{2} & \text { if no sectors contaminated } \\ 0 & \text { else. }\end{cases}
$$




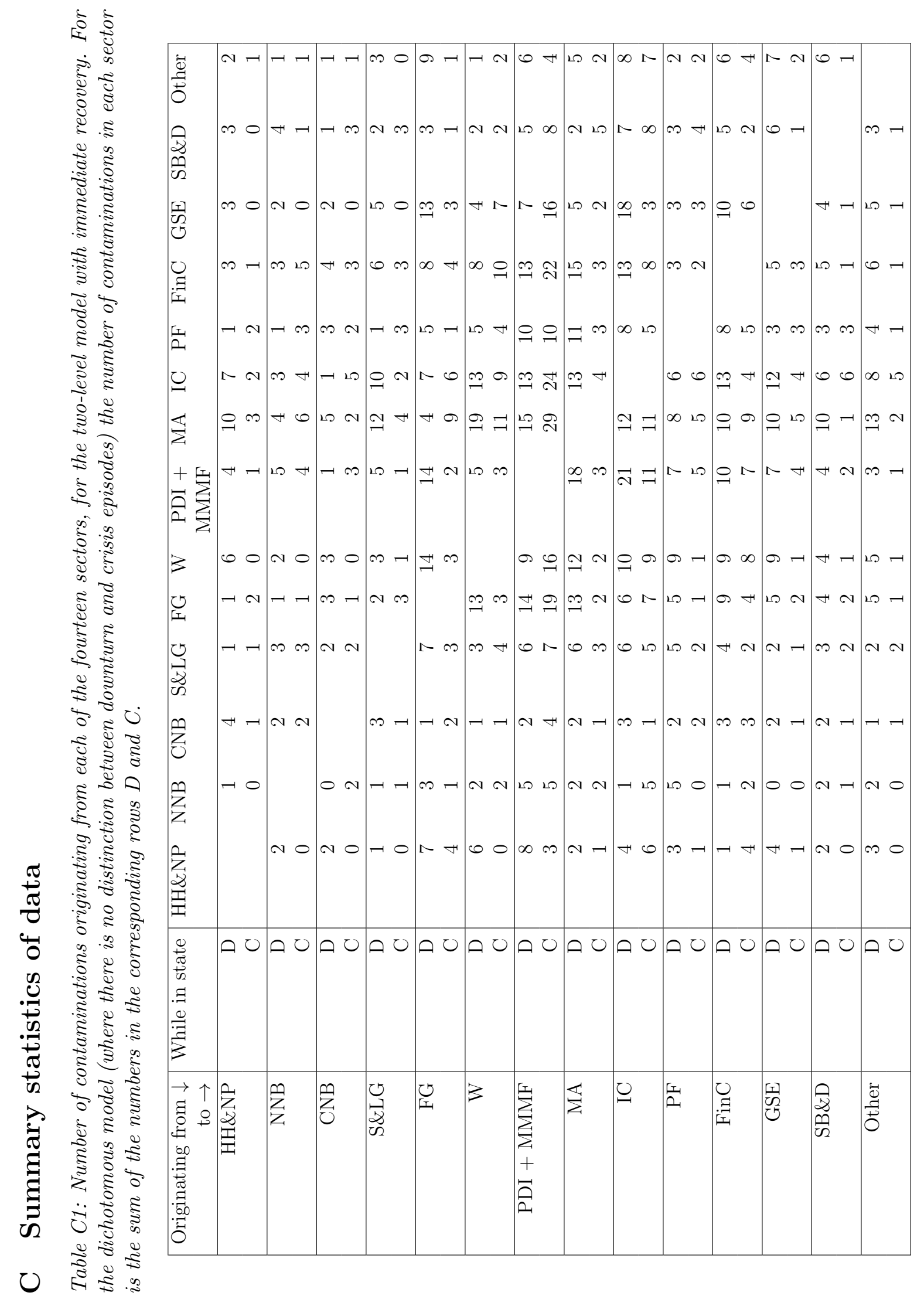




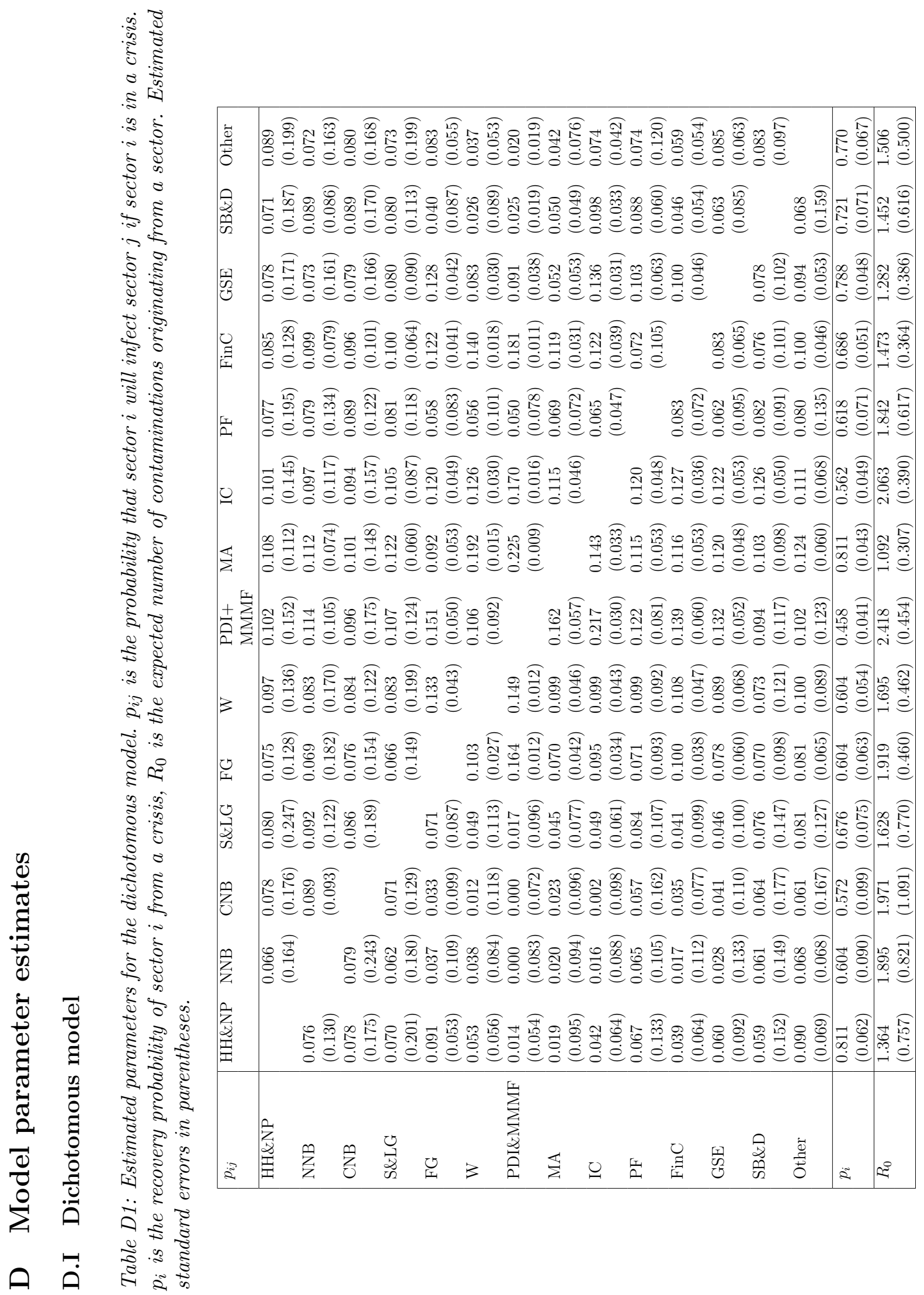




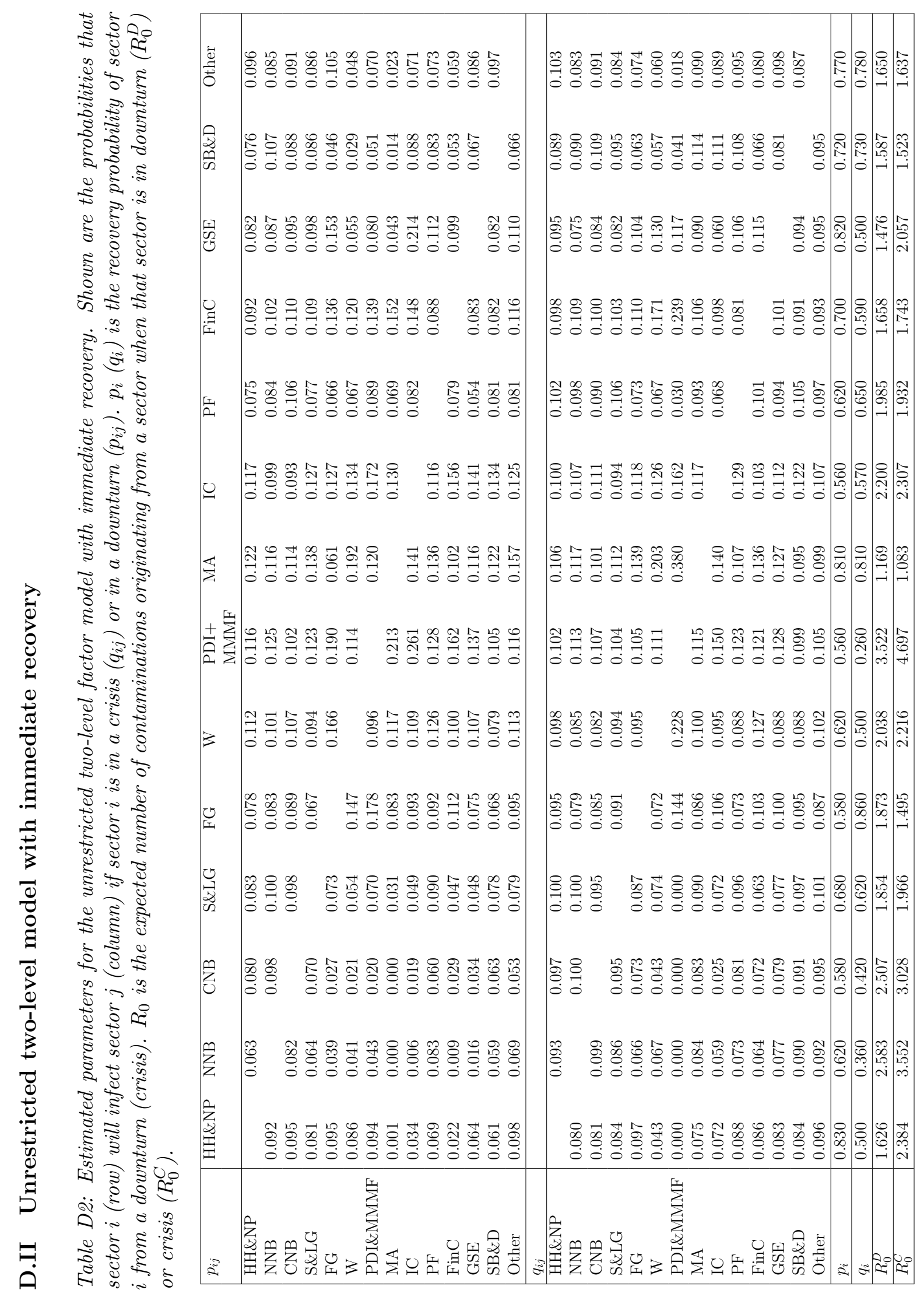




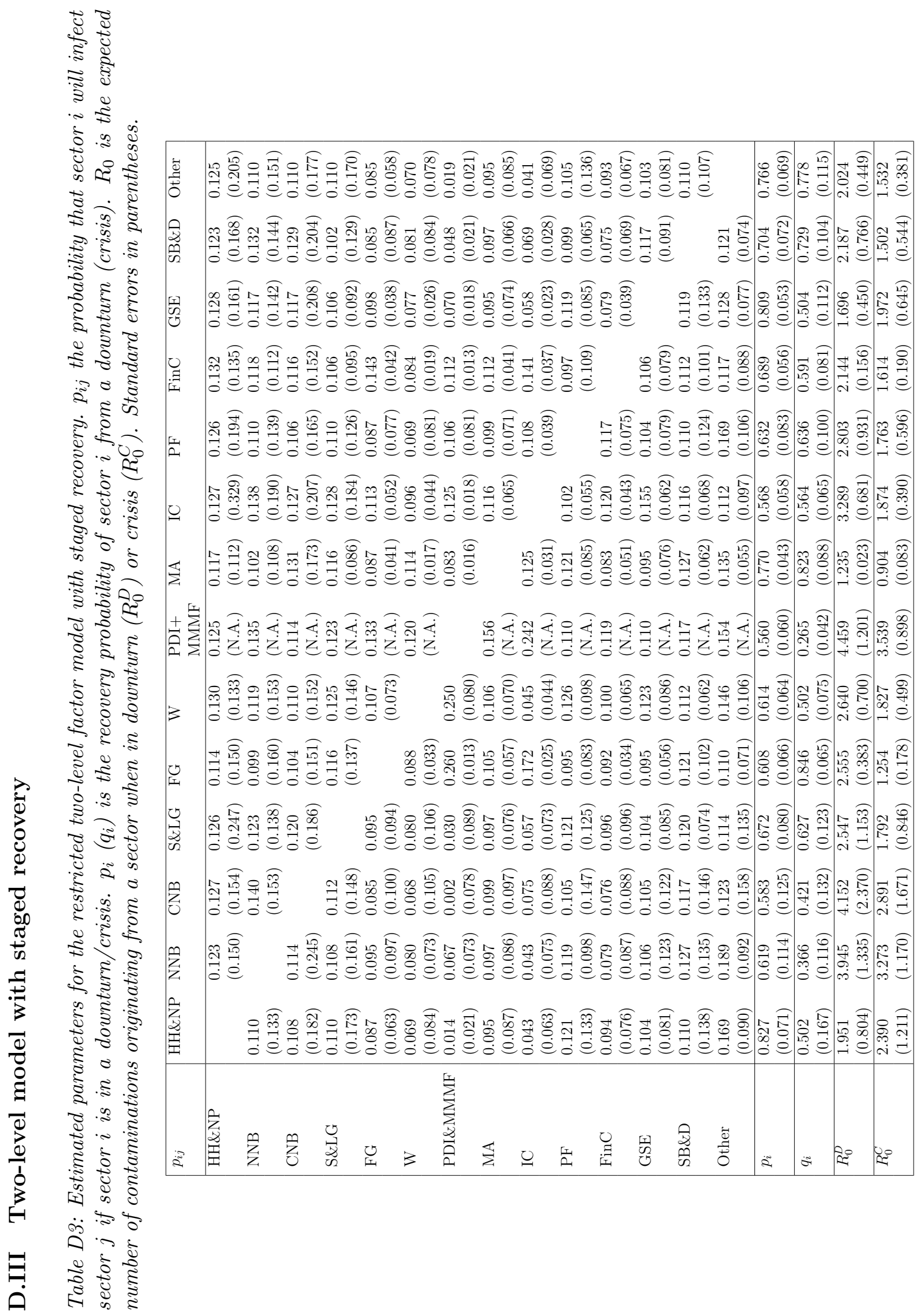




\section{E Robustness}

\section{E.I Robustness over time}

In this section, the robustness of the estimated models over time is assessed. For the dichotomous model, this is done by performing a rolling window estimation over the full time sample, as the model can be estimated fairly quickly. For the other models under consideration (that are much more computationally intensive), we will consider a sample split. Specifically, the robustness of the $R_{0}$ statistics to this split will be evaluated.

\section{Robustness of the dichotomous model over time}

In Figure E1, the time-varying reproduction number estimates $\left(R_{0}\right)$ are represented for the dichotomous model, based on a rolling window estimation, with a window length of 120 observations. As can be seen, for almost all sectors, the $R_{0}$ is remarkably stable over time. The only main exception is PDI\&MMMF, which has a large drop in infectiousness at 1986 and is lower from then on. At its most infectious period, when using the sample from 1956Q1-1986Q1, the $R_{0}$ statistic for this sector was as high as 4.15, while at later periods it only reached values around 1.6. This is not so surprising, given how the crises of this sector are spread over time, see Figure 7. On the other hand, IC has a small positive trend in infectiousness, with values of $R_{0}$ of 1.9 at the beginning of the sample and values as high as 2.5 at the end. Noticeably, it peaks at 2.67 as soon as 2007Q1 enters the rolling estimation window. 


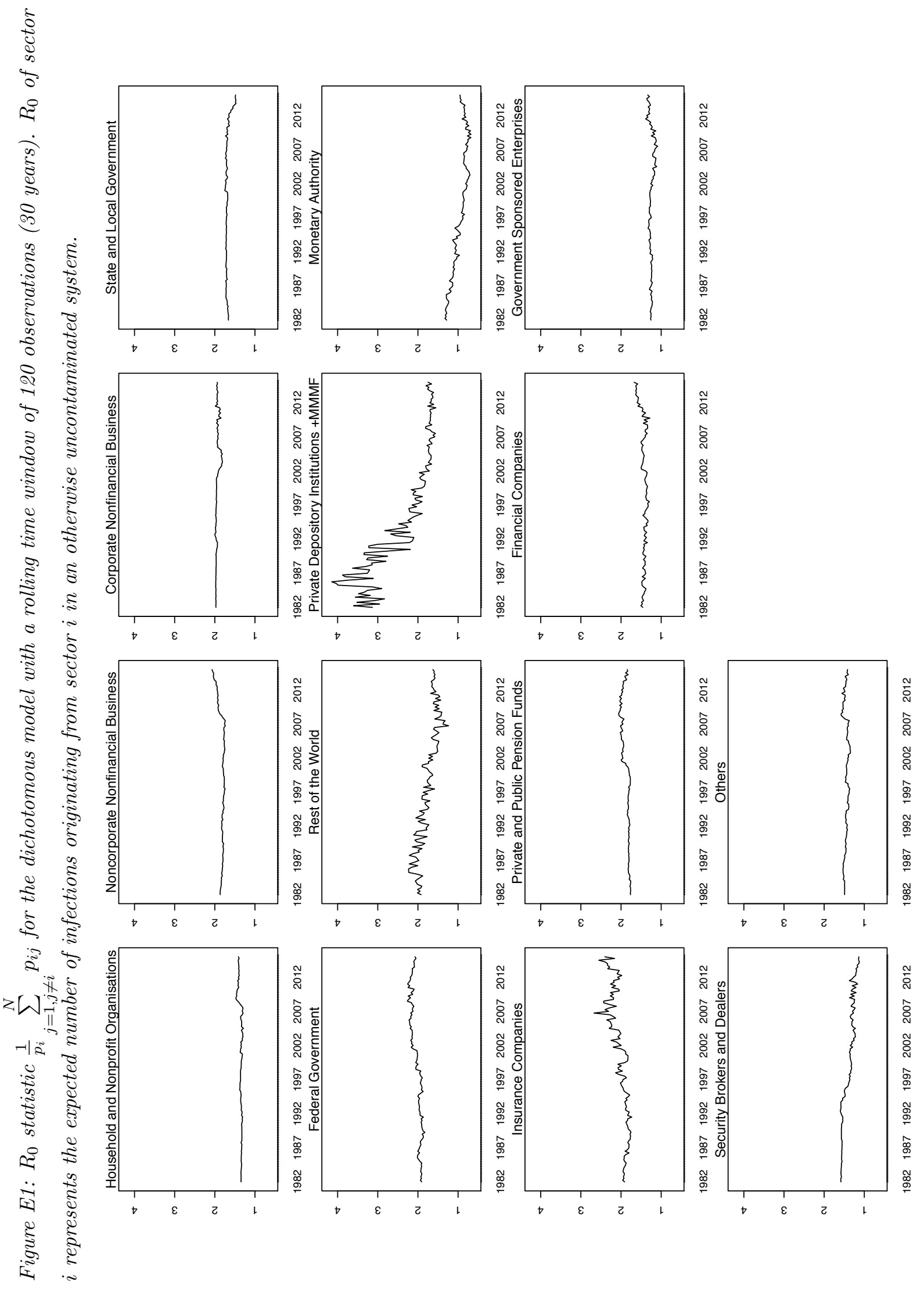




\section{Robustness of the restricted two-level model with immediate recovery over time}

In this section, the robustness of the unrestricted two-level model with immediate recovery is discussed. Specifically, we focus on the stability of the $R_{0}$ estimates over time. This is done by splitting the samples into two parts, the first sample contains the first 155 observations, the second sample contains the last 100 observations. Thus, the data is split into the period before 1990 and the period after. In Table E1, the two different $R_{0}$ estimates are supplied for both time periods.

Table E1: Expected number of contaminations originating from the specific sector when in downturn $\left(R_{0}^{D}\right)$ and when in crisis $\left(R_{0}^{C}\right)$ for the restricted two-level model with immediate recovery, data split into two subsamples, calculated using Equations 2 and 1, respectively. Standard errors (in parentheses) computed via the delta method.

\begin{tabular}{|c|c|c|c|c|}
\hline Sample period & \multicolumn{2}{|c|}{$1952-1990$} & \multicolumn{2}{|c|}{ 1990-2015 } \\
\hline Sector & (s.e.) & (s.e.) & (s.e.) & (s.e.) \\
\hline HH\&NP & $1.645(1.448)$ & $2.474(3.198)$ & $1.629(4.050)$ & $2.353(5.903)$ \\
\hline NNB & $2.539(2.124)$ & $3.394(3.164)$ & $2.791(2.959)$ & $3.924(4.323)$ \\
\hline $\mathrm{CNB}$ & $2.523(2.847)$ & $3.013(3.892)$ & $2.371(2.706)$ & $2.796(3.268)$ \\
\hline S\&LG & $1.889(1.233)$ & $2.017(1.398)$ & $1.669(1.762)$ & $1.770(1.932)$ \\
\hline FG & $1.806(0.527)$ & $1.397(0.413)$ & $1.923(1.720)$ & $1.556(1.732)$ \\
\hline W & $2.098(0.615)$ & $2.374(0.775)$ & $1.997(1.867)$ & $2.290(2.249)$ \\
\hline PDI\&MMMF & $4.150(1.282)$ & $6.231(2.025)$ & $2.353(1.727)$ & $2.895(2.203)$ \\
\hline MA & $1.298(0.463)$ & $1.322(0.496)$ & $1.082(0.709)$ & $1.019(0.778)$ \\
\hline $\mathrm{IC}$ & $1.962(0.710)$ & $1.960(0.777)$ & $2.937(0.746)$ & $2.812(0.791)$ \\
\hline $\mathrm{PF}$ & $1.939(1.212)$ & $1.917(1.311)$ & $2.031(1.490)$ & $1.872(1.406)$ \\
\hline FinC & $1.583(0.571)$ & $1.772(2.329)$ & $1.892(1.315)$ & $2.144(1.569)$ \\
\hline GSE & $1.558(0.639)$ & $2.329(1.355)$ & $1.364(1.033)$ & $1.897(1.520)$ \\
\hline SB\&D & $1.738(1.292)$ & $1.705(1.562)$ & $1.160(0.980)$ & $1.191(1.026)$ \\
\hline Other & $1.626(1.116)$ & $1.629(1.928)$ & $1.560(2.260)$ & $1.480(2.155)$ \\
\hline
\end{tabular}

Overall, Table E1 suggests that a small decrease in the contagiousness of most sectors occurred after 1990, with a few notable exceptions. First, the infectiousness of PDI\&MMMF has changed a lot over time. In the period 1952-1990, PDI\&MMMF had an overall infectiousness of $4.150\left(R_{0}^{D}\right)$, but during 1990-2015, the infectiousness decreased to 2.353. Yet the decreased infectiousness in this sector was accompanied by a decrease in the infectiousness of most other sectors so that even in the later period, PDI\&MMMF is still one of the more infectious sectors. An opposite pattern is evident for Insurance Companies, for which the values of $R_{0}$ have increased by around one full point over time; this is all the more striking given that most other sectors experienced a decline in contagiousness. For the other sectors, the changes in $R_{0}$ are a lot smaller over the two sample periods, with small decreases in both $R_{0}$ s observable for HH\&NP, CNB, S\&LG, Rest of the World, MA, GSE, SB\&D and Other. 


\section{Robustness of the restricted two-level model with staged recovery over time}

Analogous to what was done for the two-level model with immediate recovery, the sample is split into two parts (before 1990 and after) and the model is estimated for both subsamples. The estimates of $R_{0}$ are reported in Table E2.

Table E2: Expected number of contaminations originating from the specific sector when in downturn $\left(R_{0}^{D}\right)$ and when in crisis $\left(R_{0}^{C}\right)$ for the restricted two-level model with staged recovery, data split into two subsamples, calculated using Equations 5 and 3, respectively. No standard errors available, because of lack of data on some sector interactions.

\begin{tabular}{|r|cc|cc|}
\hline Sample period & \multicolumn{2}{|c|}{$1952-1990$} & \multicolumn{2}{|c|}{$1990-2015$} \\
\hline Sector & $R_{0}^{D}$ & $R_{0}^{C}$ & $R_{0}^{D}$ & $R_{0}^{C}$ \\
\hline Household and Nonprofit Organisations & 1.920 & 2.436 & 2.013 & 2.328 \\
Noncorporate Nonfinancial Business & 3.850 & 3.252 & 4.165 & 3.347 \\
Corporate Nonfinancial Business & 4.303 & 3.003 & 3.912 & 2.669 \\
State and Local Government & 2.652 & 1.881 & 2.402 & 1.677 \\
Federal Government & 2.519 & 1.203 & 2.855 & 1.432 \\
Rest of the World & 2.460 & 1.622 & 2.874 & 2.197 \\
Private Depository Institutions + MMMF & 5.839 & 4.927 & 2.809 & 1.995 \\
Monetary Authority & 1.261 & 0.998 & 1.303 & 0.833 \\
Insurance Companies & 2.909 & 1.726 & 3.776 & 1.958 \\
Private and Public Pension Funds & 2.830 & 1.813 & 2.780 & 1.670 \\
Financial Companies & 1.904 & 1.425 & 2.419 & 1.932 \\
Government Sponsored Enterprises & 1.660 & 2.046 & 1.800 & 1.907 \\
Security Brokers and Dealers & 2.327 & 1.644 & 1.854 & 1.251 \\
Other & 2.016 & 1.599 & 1.917 & 1.343 \\
\hline
\end{tabular}

Very similar patterns can be observed as in the immediate recovery model; the Private Depository Institutions and Money Market Mutual Funds sector experienced strong decreases in infectiousness and the Insurance Companies sector had a strong increase in $R_{0}$. For Insurance Companies the increase in $R_{0}^{D}$ is especially large, indicating that the expected number of sectors it will infect from the moment it enters a downturn is much higher. On closer inspection of the estimated parameters that enter the computation of $R_{0}$ (i.e., each of the probabilities from the Insurance Companies sector to each of the other sectors), it is clear that the explanation of this increase in infectiousness lies in an increase in the infection probabilities of the following sectors: PDI\&MMMF, PF, GSE and SB\&D. The $R_{0}$ of Financial Companies has increased as well, by around 0.5 (that is, on average an additional half a sector is infected by either a downturn or a crisis in this sector). 


\section{E.II Robustness to the crisis definition}

To assess the robustness of the results with respect to the crisis definitions used, in this section, an alternative crisis definition is introduced. Specifically, we define sector-specific crisis thresholds, such that every sector is in an infectious state exactly $25 \%$ of the time. The motivation for this definition is to balance the number of "crises" across the different sectors, for example because some sectors might historically have lower steady-state growth and hence would need more severe negative growth to be considered in crisis than sectors that have always had positive growth. Although the alternative as we specify it is a fullsample definition, it should be obvious that it can easily be adapted to be dynamic (e.g., define the threshold to be the level of growth that represents the bottom quartile of growth rates for that sector over the previous ten years). The different states of the fourteen sectors over time according to this alternative definition are visualized in Figure E2. The biggest difference is visible when looking at PDI\&MMMF, which now experiences a lot fewer crises. Although unconditionally having fewer crises is likely to cause a sector to be less infectious than it was under the previous crisis definition (and similarly, having more crises will likely cause a sector to be more infectious), when considering infection transmission this will not always be the case, because it depends if the remaining episodes are followed by more crises (according to the new definition) in the other sectors.

In Table E3, the estimates of the expected number of contaminations, $R_{0}$, are given for this alternative definition of a crisis when applying the restricted two-level model with immediate recovery. We can see that there are a lot of similarities between these $R_{0}$ estimates and the $R_{0}$ estimates of Table 6 . When studying $R_{0}^{D}$, the expected number of contaminations once in a downturn, it can be seen that the $R_{0}^{D}$ of both CNB and NNB are still very high and in fact, the order of magnitude remains comparable. Under the previous crisis definition, PDI\&MMMF was the most infectious sector, now it is the third most infectious sector according to $R_{0}^{C}$ (infectiousness once in a crisis) and the fourth most infectious according to $R_{0}^{D}$. The monetary authority (MA) remains the least infectious sector.

In Table E4, the estimates of the contamination probabilities and recovery probabilities are reported. These estimates can be compared to the estimates under the original crisis definition, given in Table 5. We can observe that PDI\&MMMF is now more likely to recover when in a downturn and less likely to infect W, IC, GSE, FinC and FG. The probability of PDI\&MMMF to infect monetary authority has also decreased, but is still very much significantly different from zero. Most of the other estimates are very comparable to the ones found under the previous crisis definition. 
Figure E2: Different states of the fourteen sectors over time, according to the definition of the immediate recovery models, where every sector is in a downturn or crisis $25 \%$ of the time. The light color refers to the susceptible state, while the darker color to a crisis.

\section{Development of crises over time}

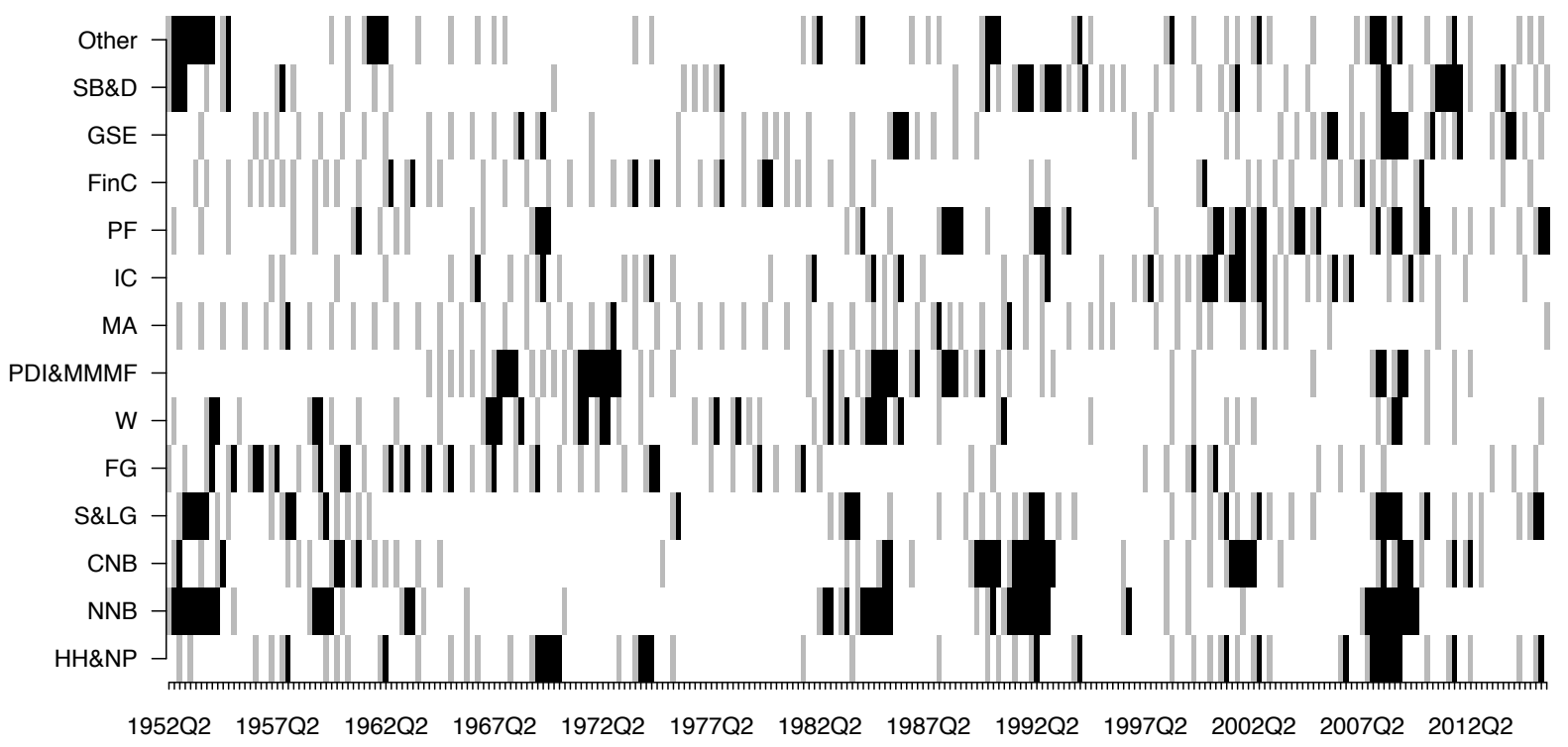

Table E3: Expected number of contaminations originating from the specific sector when in downturn $\left(R_{0}^{D}\right)$ and when in crisis $\left(R_{0}^{C}\right)$ for the restricted two-level model with immediate recovery and sectorspecific threshold, calculated using Equations 2 and 1, respectively. Standard errors (in parentheses) computed via the delta method.

\begin{tabular}{|r|ccc|}
\hline Sector & $R_{0}^{D}$ & (s.e.) & $R_{0}^{C}($ s.e. $)$ \\
\hline Household and Nonprofit Organisations & $1.691(0.643)$ & $2.262(0.948)$ \\
Noncorporate Nonfinancial Business & $2.775(1.035)$ & $3.903(1.545)$ \\
Corporate Nonfinancial Business & $2.302(0.820)$ & $2.818(1.094)$ \\
State and Local Government & $1.543(0.749)$ & $1.744(0.876)$ \\
Federal Government & $1.514(0.503)$ & $1.195(0.404)$ \\
Rest of the World & $1.570(0.664)$ & $1.762(0.795)$ \\
Private Depository Institutions + MMMF & $1.781(0.933)$ & $2.699(1.513)$ \\
Monetary Authority & $0.972(0.406)$ & $1.037(0.630)$ \\
Insurance Companies & $1.746(0.572)$ & $1.760(0.646)$ \\
Private and Public Pension Funds & $1.615(0.638)$ & $1.602(0.654)$ \\
Financial Companies & $1.461(0.464)$ & $1.719(0.719)$ \\
Government Sponsored Enterprises & $1.169(0.460)$ & $1.735(0.789)$ \\
Security Brokers and Dealers & $1.350(0.497)$ & $1.396(0.532)$ \\
Other & $1.882(0.584)$ & $1.984(0.638)$ \\
\hline
\end{tabular}




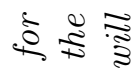

की

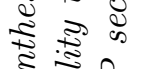

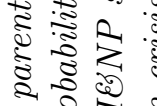

ह हू,

$\stackrel{5}{\infty} \approx$

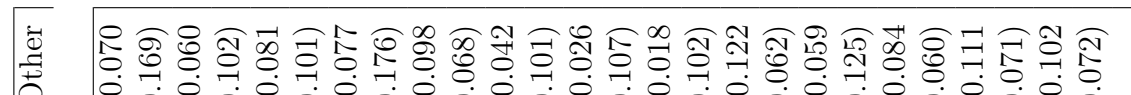

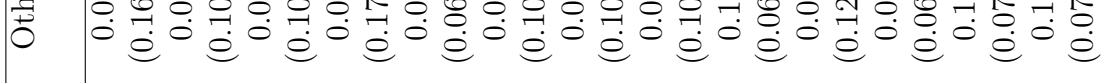

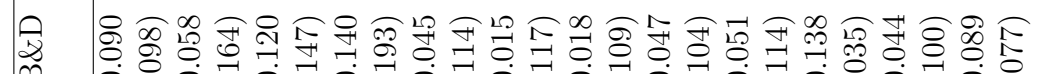

w 0 过

㲁

등ํㅇ영

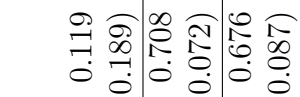

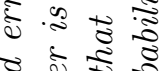

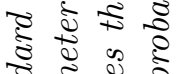

-

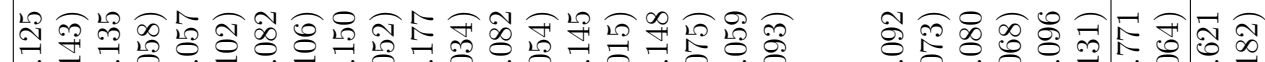

동ㅇำ

茫

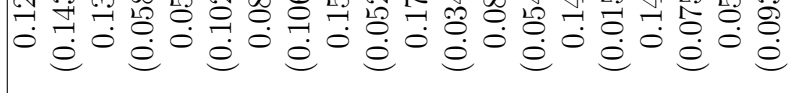

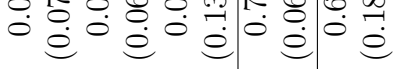

$\stackrel{1}{a}$

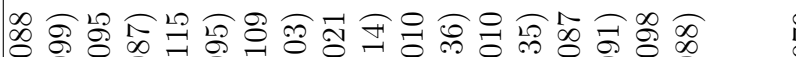

앙

권

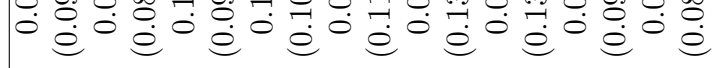

0

응

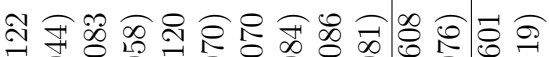

- क व

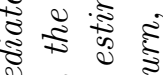

हैं

हो क क

莡

จ. ह है है

๑

है 2 :

कृ

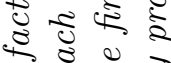

ये

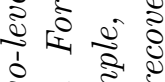

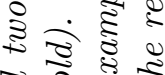

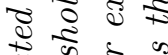

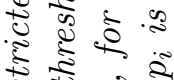

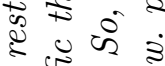

๘

i.

क को

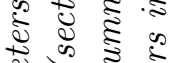

ह 8

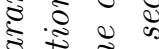

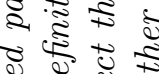

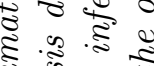

के

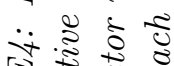

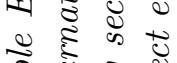

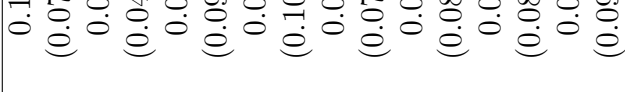

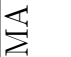

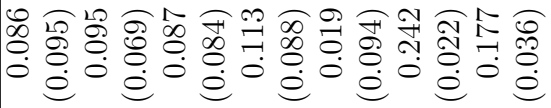

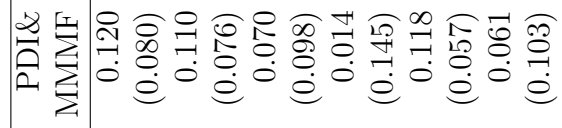

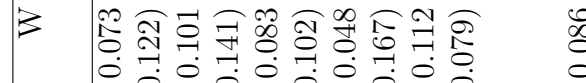

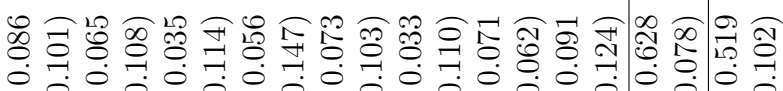

U

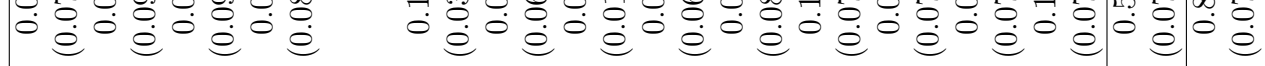

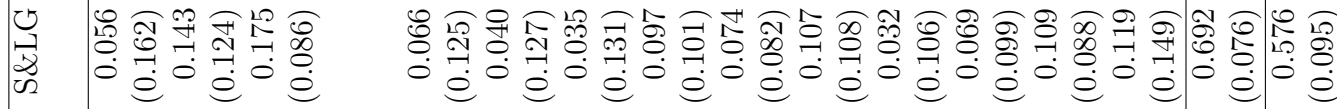

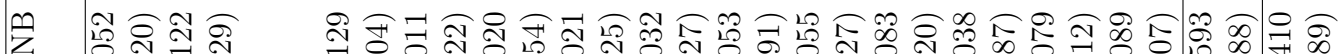

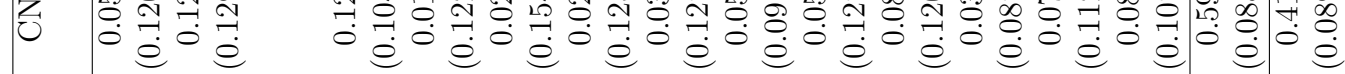

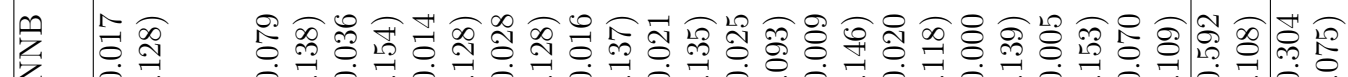

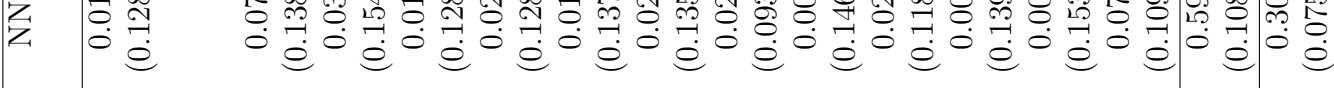

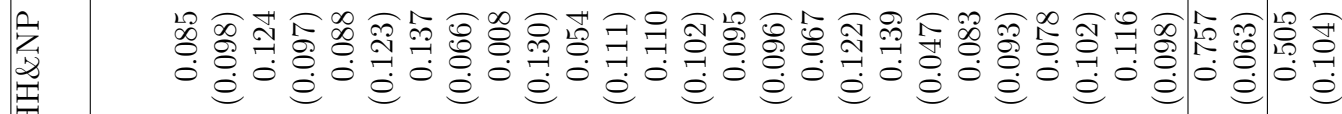

田

蓄

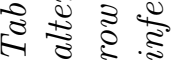




\section{E.III Robustness to seasonal variation}

Because the analysis uses non-seasonally-adjusted data, it is possible that some of the observed net worth growth declines that are labeled as a downturn or crisis are seasonal fluctuations that would not be particularly worrying to a policymaker. To account for this possibility, we evaluate the robustness of our results when we first deseasonalize the data by regressing each sectors net worth growth on a set of quarterly dummy variables and using the residuals from this regression to identify periods of negative (deseasonalized) net worth growth. The resultant deseasonalized series is hence net worth growth with quarterly means taken out.

The different states of the fourteen sectors over time using the deseasonalized net worth growth data are visualized in Figure E3. Comparing this figure with Figure 7, it can be seen that for many sectors, there is little change, indicating little difference in the quarterly means in a series. For the Monetary Authority sector and 'Other', however, we do observe changes in the pattern of downturns and crises.

In Table E5, the estimates of the expected number of contaminations, $R_{0}$, are given for the downturn/crisis data based on deseasonalized net worth growth rates. Most $R_{0}$ estimates remain very close to the estimates in Table 6 . The biggest difference is a decline in the estimated number of contaminations that result from a downturn or crisis of PDI\&MMMF, although even after deseasonalizing it remains one of the most infectious sectors. Before deseasonalizing the net worth growth rates, the expected number of contaminations originating from PDI\&MMMF when in a crisis, $R_{0}^{C}$, had a value of approximately 5.2, while the seasonal adjustment reduces this number to 3.2. Similarly, the expected number of contaminations originating from PDI\&MMMF when in a downturn, $R_{0}^{D}$, declines from 3.6 to 2.5. Notably, despite the difference between Figure E3 and Figure 7 suggesting that the net worth growth of MA has a strong seasonal component, the expected number of contaminations of the MA sector is hardly affected by this particular choice of deseasonalization. Thus these specific results do not appear to be driven by seasonal patterns in the data.

In order to better understand what may be driving the changes in $R_{0}$, in Table E6 the estimates of the contamination probabilities and recovery probabilities are reported. These can be compared with the estimates for the unadjusted data, that is, Table 5. First focusing on PDI\&MMMF, it can be seen that the probability of recovering from a crisis increases from 0.258 to 0.315 , contributing to PDI\&MMMF being less infectious overall. The recovery probabilities for all other sectors remain almost unaffected by the seasonal adjustment. 
Furthermore, the contamination probabilities of PDI\&MMMF to other sectors decrease for almost all sectors, explaining the overall decreased infectiousness of PDI\&MMMF. For example, the probability of infecting MA declines from 0.29 to 0.17 . Only the probability of infecting SB\&D increases, from 0.030 to 0.117 . From this robustness exercise, we know that at least part of the estimated infectiousness of PDI\&MMMF can be attributed to seasonal fluctuations. One other notable change is that the MA sector is less likely to be infected by all other sectors, with the biggest declines coming from transmissions from the PDI\&MMMF and $\mathrm{W}$ sectors.

Despite the above-mentioned changes after seasonal adjustment to the data, most visibly in the PDI\&MMMF sector, the overall inference remains qualitatively the same. PDI\&MMMF, NNB and CNB remain the most infectious sectors and MA is the least infectious sector. Also quite infectious is the IC sector.

Figure E3: Different states of the fourteen sectors over time, according to the definition of the immediate recovery models, where the net worth growth rates have been deseasonalized via regression on quarterly dummy variables. The light color refers to the susceptible state (downturn), while the darker color refers to a crisis.

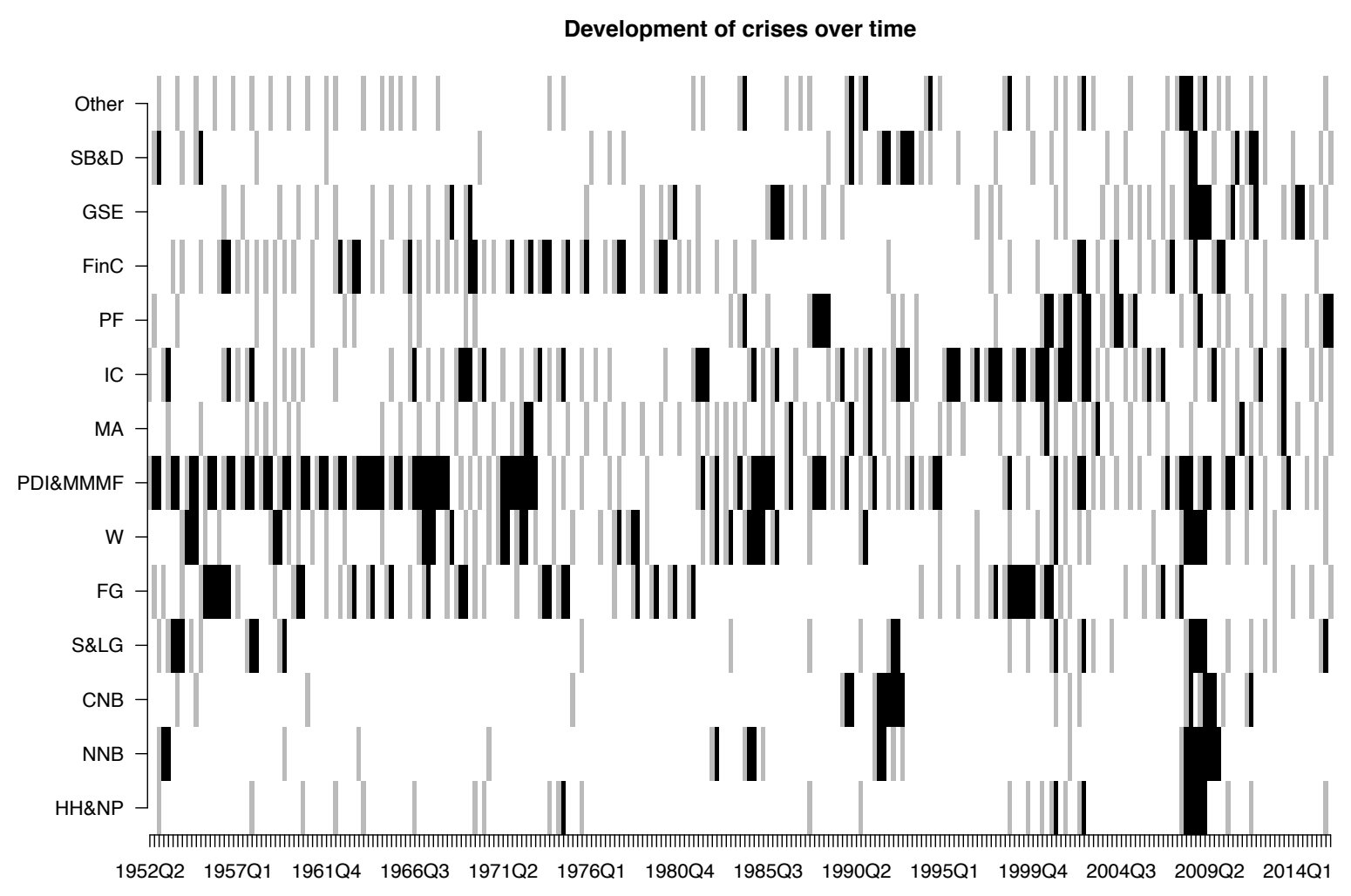


Table E5: Expected number of contaminations originating from the specific sector when in downturn $\left(R_{0}^{D}\right)$ and when in crisis $\left(R_{0}^{C}\right)$ for the restricted two-level model with immediate recovery and deseasonalized net worth growth rates, calculated using Equations 2 and 1, respectively. Standard errors (in parentheses) computed via the delta method.

\begin{tabular}{|r|ccc|}
\hline Sector & $R_{0}^{D}$ & $($ s.e. $)$ & $R_{0}^{C}($ s.e. $)$ \\
\hline Household and Nonprofit Organisations & $1.592(1.065)$ & $2.374(1.761)$ \\
Noncorporate Nonfinancial Business & $2.438(1.682)$ & $3.294(2.402)$ \\
Corporate Nonfinancial Business & $2.428(1.756)$ & $2.908(2.211)$ \\
State and Local Government & $1.786(0.985)$ & $1.911(1.095)$ \\
Federal Government & $1.676(0.485)$ & $1.368(0.394)$ \\
Rest of the World & $1.832(0.620)$ & $2.111(0.775)$ \\
Private Depository Institutions + MMMF & $2.541(0.693)$ & $3.232(0.930)$ \\
Monetary Authority & $1.241(0.399)$ & $1.266(0.467)$ \\
Insurance Companies & $2.319(0.446)$ & $2.269(0.493)$ \\
Private and Public Pension Funds & $1.939(0.674)$ & $1.947(0.728)$ \\
Financial Companies & $1.594(0.490)$ & $1.763(0.598)$ \\
Government Sponsored Enterprises & $1.513(0.490)$ & $2.206(0.879)$ \\
Security Brokers and Dealers & $1.552(0.682)$ & $1.558(0.712)$ \\
Other & $1.487(0.594)$ & $1.490(0.638)$ \\
\hline
\end{tabular}




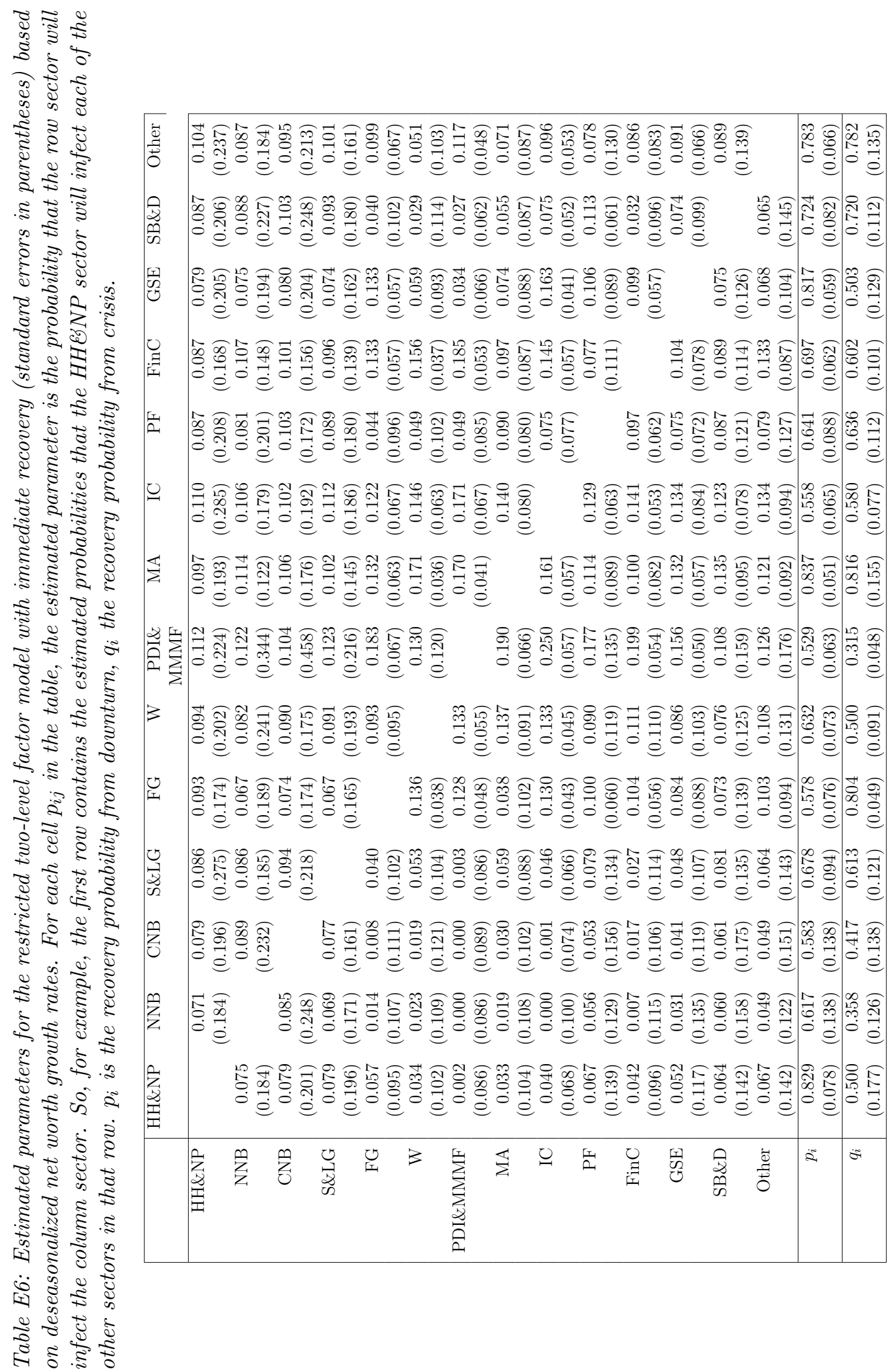

